\title{
ANALYSIS OF A FINITE VOLUME ELEMENT METHOD FOR THE STOKES PROBLEM
}

\author{
ALFIO QUARTERONI ${ }^{\dagger, \ddagger}$ AND RICARDO RUIZ BAIER ${ }^{\dagger}$
}

\begin{abstract}
In this paper we propose a stabilized conforming finite volume element method for the Stokes equations. On stating the convergence of the method, optimal a priori error estimates in different norms are obtained by establishing the adequate connection between the finite volume and stabilized finite element formulations. A superconvergence result is also derived by using a postprocessing projection method. The stabilization of the continuous lowest equal order pair finite volume element discretization $\left(\mathbb{P}_{1}-\mathbb{P}_{1}\right)$ is achieved by enriching the velocity space with bubble-like functions. Finally, some numerical experiments that confirm the predicted behavior of the method are provided.
\end{abstract}

\section{INTRODUCTION}

Finite volume element methods (FVEM) [6], also known as marker and cell methods, generalized difference methods 24, finite volume methods 23, 32, covolume methods [7] or box methods 3, 11, are approximation methods that could be placed somehow in between classical finite volume schemes and standard finite element (FE) methods. Roughly speaking, the FVEM is able to keep the simplicity and conservativity of finite volume methods and at the same time permits a natural development of error analysis in the $L^{2}$-norm as in standard FE methods. This is basically achieved by introducing a transfer map which allows to rewrite the FE formulation as its finite-volume-like counterpart, i.e., using piecewise constant test functions. The usual difficulty in the analysis of finite volume methods consists in that trial and test functions lie in different spaces and are associated with different meshes. In the FVEM approach, a complementary dual mesh is also constructed, and this is commonly done by connecting the barycenters of the triangles in the FE mesh, with the midpoints of the associated edges (see [7, 10, 20, 23]). However, one of the most appealing features is that the approximate solution is found in the same subspace used in the construction of the FE method. In fact, FVE methods might be regarded as a special class of Petrov-Galerkin methods where the trial function spaces are connected with the test functions' spaces associated with the dual partition induced by the control volumes [21, 23. Moreover, the approach used herein (based on the relation between finite volume and FE approximations) possesses the appealing feature of being locally conservative.

As for the numerical approximation of Stokes equations, numerous methods have been proposed, analyzed and tested (for an overview, the reader is referred to 18 and the references therein). In the framework of finite volume methods, recent contributions include the work by Gallouët et al. 17] which treat the nonlinear case based on Crouzeix-Raviart elements, Nicase and Djadel [25] prove different error estimates for a finite volume scheme by using nonconforming elements, Eymard et al. [14] obtained error estimates for a stabilized finite volume scheme based on the Brezzi-Pitkäranta method. Regarding FVE approximations for the Stokes problem, in his early paper, Chou [7] used nonconforming piecewise linear elements for velocity and piecewise

Date: Received: date

Updated: March 15, 2010.

1991 Mathematics Subject Classification. 65N30, 35Q30, 65N12, 65N15.

Key words and phrases. Stokes problem, multiscale stabilization, finite volume element method, a priori error estimates, superconvergence analysis.

${ }^{\dagger}$ CMCS-MATHICSE-SB, Ecole Polytechnique Fédérale de Lausanne EPFL, Station 8, CH-1015, Lausanne, Switzerland. Email: ricardo.ruiz@epfl.ch.

${ }^{\ddagger}$ MOX - Dipartimento di Matematica F. Brioschi, Politecnico di Milano via Bonardi 9, 20133 Milano, Italy.

Email: alfio.quarteroni@epfl.ch. 
constant for pressure. In the contribution by Ye [30, the analysis is carried out for both conforming and nonconforming elements on triangles and rectangles. We also mention the recent work of $\mathrm{Li}$ and Chen [23] who advanced a FVE method based on a stabilization method that uses the residual of two local Gauss integration formulae on each finite element.

In this paper we will devote ourselves to the study of a particular stabilized FVE method constructed on the basis of a conforming finite element formulation where the velocity and pressure fields are approximated by piecewise linear polynomials. Since the considered approximation of the Stokes equations is based on the pair $\mathbb{P}_{1}-\mathbb{P}_{1}$ that does not satisfy the discrete inf-sup condition (see [18]), one of the most common remedies consists in including a stabilization technique, i.e., to add a mesh dependent term to the usual formulation. One of the motivations for keeping the unstable pair of lowest equal order elements, is that they allow a more efficient implementation, by achieving a reduction of the number of unknowns in the final systems. Among the wide class of stabilized FE formulations available from the literature, such as Streamline-Upwind/PetrovGalerkin (SUPG), Galerkin-Least-Squares (GLS) and other methods (see for instance [27, Sect. 9.4] and the references therein), in this paper we include a stabilization technique similar to the one introduced by Franca et al. [16, in which a Petrov-Galerkin approach is used to enrich the trial space with bubble functions being solutions to a local problem involving the residual of the momentum equation, which can be solved analytically. As recently proposed by Araya et al. 2, by enriching the velocity space using a multiscale approach combined with static condensation, the resulting FE method includes the classical GLS additional terms at the element level and a suitable jump term on the normal derivative of the velocity field at the element boundaries. For the latter, the stabilization parameter is known exactly.

For our method, the essential point is to appropriately connect the FE and FVE formulations. After establishing such relationship we deduce the corresponding optimal a priori error estimates for the new stabilized FVE method using a usual approach for classical FE methods. In contrast to classical finite volume schemes, the velocity fluxes will not be discretized in a finite-difference fashion. This fact plays an important role at the implementation stage as well, since all the information corresponding to the dual partition, needed for the derivation of the FVE formulation can be retrieved from the information on the edges of the primal mesh.

Another important novel ingredient of this paper is the superconvergence analysis of the approximate solution. The main goal is to improve the current accuracy of the approximation by applying a postprocessing technique constructed on the basis of a projection method presented in [19, 29, 22, 31. Super-convergence properties of FVE approximations in the nonconforming and conforming cases were first studied in the recent works by Cui and Ye [10] and Wang and Ye [29]. The technique consists in projecting the FVE space to another approximation space (possibly of higher order) related to a coarser mesh. A detailed study including the analysis of a posteriori error estimates for FVE methods in the spirit of [5, 12, and adaptivity following [8] have been postponed for a forthcoming paper. Further efforts are also being made to extend the analysis herein presented to the transient Navier-Stokes equations.

The remainder of the paper is organized as follows. In the next section, a set up of some preliminary results and notations concerning the spaces involved in the analysis is followed by a detailed description of the model problem and the FE discretization used as reference. Further, some auxiliary lemmas are also provided in that section. Next, the stabilized finite volume formulation that we will employ and its corresponding link with the reference finite element method are provided in Section 3 . The main results of the paper, namely the convergence analysis of the stabilized finite volume element approximation, are proved in Section 4 and additional superconvergence estimates are given in Section 5 . Finally, Section 6 is devoted to the presentation of an illustrative numerical test which confirms the expected rates of convergence and superconvergence. 


\section{Preliminaries}

The standard notation will be used for Lebesgue spaces $L^{p}(\Omega), 1 \leq p \leq \infty, L_{0}^{2}(\Omega)=\left\{v \in L^{2}(\Omega)\right.$ : $\left.\int_{\Omega} v=0\right\}$ and Sobolev functional spaces $H^{m}(\Omega), H_{0}^{1}(\Omega)=\left\{v \in H^{1}(\Omega): v=0\right.$ on $\left.\partial \Omega\right\}$, where $\Omega$ is an open, bounded and connected subset of $\mathbb{R}^{2}$ with polygonal boundary $\partial \Omega$. Further, let us denote $\boldsymbol{H}^{m}(\Omega)=H^{m}(\Omega)^{2}$, and in general $\boldsymbol{M}$ will denote the corresponding vectorial counterpart of the scalar space $M$. For a subset $R \subset \Omega,(\cdot, \cdot)_{R}$ denotes the $L^{2}(R)$-inner product. In addition, $\mathbb{P}_{r}(R)$ will represent the space of polynomial functions of degree $s \leq r$ on $R$.

2.1. The Boundary Value Problem. Let us consider the following steady state Stokes problem with Dirichlet boundary conditions: Find $\boldsymbol{u}, p$ such that

$$
\begin{aligned}
-\nu \Delta \boldsymbol{u}+\nabla p & =\boldsymbol{f} & & \text { in } \Omega, \\
\nabla \cdot \boldsymbol{u} & =0 & & \text { in } \Omega, \\
\boldsymbol{u} & =\mathbf{0} & & \text { on } \partial \Omega .
\end{aligned}
$$

This linear problem describes the steady motion of an incompressible viscous fluid. As usual, the sought quantities are the vectorial velocity field $\boldsymbol{u}$, the scalar pressure $p$, the prescribed external force $\boldsymbol{f}$ and the constant fluid viscosity $\nu>0$. Multiplying (2.1) by a test function $\boldsymbol{v},(2.2)$ by a test function $q$, integrating by parts both equations over $\Omega$ and summing the result, one obtains the weak formulation of problem (2.1)-(2.3): Find $(\boldsymbol{u}, p) \in \boldsymbol{H}_{0}^{1}(\Omega) \times L_{0}^{2}(\Omega)$ such that

$$
\nu(\nabla \boldsymbol{u}, \nabla \boldsymbol{v})_{\Omega}-(p, \nabla \cdot \boldsymbol{v})_{\Omega}+(q, \nabla \cdot \boldsymbol{u})_{\Omega}=(\boldsymbol{f}, \boldsymbol{v})_{\Omega} \quad \forall(\boldsymbol{v}, q) \in \boldsymbol{H}_{0}^{1}(\Omega) \times L_{0}^{2}(\Omega) .
$$

This model problem is well-posed (see e.g. [18] for details on the analysis).

Throughout the paper, $C>0$ will denote a constant depending only on the data $(\nu, \Omega, f)$ and not on the discretization parameters.

2.2. Finite Element Approximation. Let $\mathcal{T}_{h}$ be a regular triangulation of $\Omega$ constructed by closed triangle elements $K$ with boundary $\partial K$. We fix the numbering $s_{j}, j=1, \ldots, N_{h}$ of all nodes or vertices of $\mathcal{T}_{h}$. With $\mathcal{E}_{h}$ we denote the set of edges of $\mathcal{T}_{h}$, while $\mathcal{E}_{h}^{\text {int }}$ will denote the edges of $\mathcal{T}_{h}$ that are not part of $\partial \Omega$. In addition, $h_{K}$ denotes the diameter of the element $K$, and the mesh parameter is given by $h=\max _{K \in \mathcal{T}_{h}}\left\{h_{K}\right\}$. By $\mathcal{V}_{h}$ and $Q_{h}$ we will denote the standard linear finite element spaces for the approximation of velocity and pressure on the triangulation $\mathcal{T}_{h}$, respectively. These spaces are defined as

$$
\mathcal{V}_{h}=\left\{\boldsymbol{v} \in \boldsymbol{H}_{0}^{1}(\Omega) \cap \boldsymbol{C}^{0}(\bar{\Omega}):\left.\boldsymbol{v}\right|_{K} \in \mathbb{P}_{1}(K)^{2} \text { for all } K \in \mathcal{T}_{h}\right\}
$$

provided with the basis $\left\{\phi_{j}\right\}_{j}$, and

$$
Q_{h}=\left\{q \in L_{0}^{2}(\Omega) \cap C^{0}(\bar{\Omega}):\left.q\right|_{K} \in \mathbb{P}_{1}(K) \text { for all } K \in \mathcal{T}_{h}\right\}
$$

It is well known that with this choice of FE spaces, the classic Galerkin formulation of the problem: Find $\left(\boldsymbol{u}_{h}, p_{h}\right) \in \mathcal{V}_{h} \times Q_{h}$ such that

$$
\nu\left(\nabla \boldsymbol{u}_{h}, \nabla \boldsymbol{v}_{h}\right)_{\Omega}-\left(p_{h}, \nabla \cdot \boldsymbol{v}_{h}\right)_{\Omega}+\left(q_{h}, \nabla \cdot \boldsymbol{u}_{h}\right)_{\Omega}=\left(\boldsymbol{f}, \boldsymbol{v}_{h}\right)_{\Omega} \quad \forall\left(\boldsymbol{v}_{h}, q_{h}\right) \in \mathcal{V}_{h} \times Q_{h},
$$

does not satisfy the discrete inf-sup condition. To overcome this difficulty, we include a stabilization correction similar to that introduced in [2. In that paper, and differently than other stabilization techniques available, the stabilization parameter corresponding to the jump terms is known. Moreover, the trial velocity space is enriched with a function that does not vanish on the element boundary, which is split into a bubble part and an harmonic extension of the boundary condition. An essential point in our analysis is based on one of the formulations presented in [2]. The main ingredients of that idea are included here for sake of completeness. 


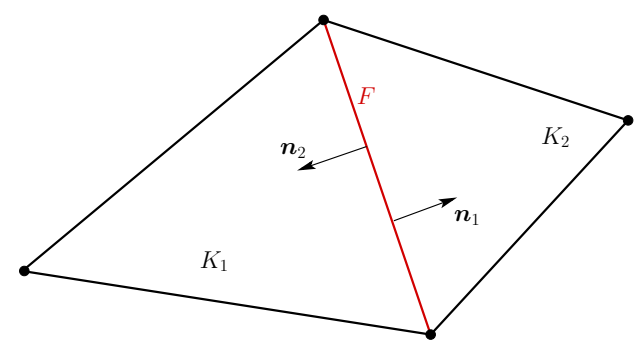

FiguRE 1. Two neighboring elements $K_{1}, K_{2} \in \mathcal{T}_{h}$ (with outer normals $\boldsymbol{n}_{1}, \boldsymbol{n}_{2}$ ) sharing the edge $F \in \mathcal{E}_{h}^{\text {int }}$.

Let $\boldsymbol{H}^{1}\left(\mathcal{T}_{h}\right)$ denote the space of functions whose restriction to $K \in \mathcal{T}_{h}$ belongs to $\boldsymbol{H}^{1}(K)$, and $\boldsymbol{E}_{h} \subset$ $\boldsymbol{H}^{1}\left(\mathcal{T}_{h}\right)$ be a finite dimensional space, called multiscale space such that $\boldsymbol{E}_{h} \cap \mathcal{V}_{h}=\{\boldsymbol{0}\}$, and consider the following Petrov-Galerkin formulation: Find $\left(\boldsymbol{u}_{h}+\boldsymbol{u}_{e}, p_{h}\right) \in\left[\mathcal{V}_{h} \oplus \boldsymbol{E}_{h}\right] \times Q_{h}$ such that

$$
\nu\left(\nabla\left(\boldsymbol{u}_{h}+\boldsymbol{u}_{e}\right), \nabla \boldsymbol{v}\right)_{\Omega}-\left(p_{h}, \nabla \cdot \boldsymbol{v}\right)_{\Omega}+\left(q_{h}, \nabla \cdot\left(\boldsymbol{u}_{h}+\boldsymbol{u}_{e}\right)\right)_{\Omega}=(\boldsymbol{f}, \boldsymbol{v})_{\Omega},
$$

for all $\left(\boldsymbol{v}, q_{h}\right) \in\left[\mathcal{V}_{h} \oplus \boldsymbol{E}_{h}^{0}\right] \times Q_{h}$, where $\boldsymbol{E}_{h}^{0}$ denotes the space of functions in $\boldsymbol{H}^{1}\left(\mathcal{T}_{h}\right)$ whose restriction to $K \in \mathcal{T}_{h}$ belongs to $\boldsymbol{H}_{0}^{1}(K)$. Notice that trial and test function spaces do not coincide. The Petrov-Galerkin scheme above can be equivalently written as: Find $\left(\boldsymbol{u}_{h}+\boldsymbol{u}_{e}, p_{h}\right) \in\left[\mathcal{V}_{h} \oplus \boldsymbol{E}_{h}\right] \times Q_{h}$ such that

$$
\begin{aligned}
& \nu\left(\nabla\left(\boldsymbol{u}_{h}+\boldsymbol{u}_{e}\right), \nabla \boldsymbol{v}_{a}\right)_{\Omega}-\left(p_{h}, \nabla \cdot \boldsymbol{v}_{a}\right)_{\Omega}+\left(q_{h}, \nabla \cdot\left(\boldsymbol{u}_{h}+\boldsymbol{u}_{e}\right)\right)_{\Omega}=\left(\boldsymbol{f}, \boldsymbol{v}_{a}\right)_{\Omega}, \\
& \nu\left(\nabla\left(\boldsymbol{u}_{h}+\boldsymbol{u}_{e}\right), \nabla \boldsymbol{v}_{b}\right)_{K}-\left(p_{h}, \nabla \cdot \boldsymbol{v}_{b}\right)_{K}=\left(\boldsymbol{f}, \boldsymbol{v}_{b}\right)_{K},
\end{aligned}
$$

for all $\boldsymbol{v}_{a} \in \mathcal{V}_{h}, q_{h} \in Q_{h}, \boldsymbol{v}_{b} \in \boldsymbol{H}^{1}(K), K \in \mathcal{T}_{h}$. Since for every $K \in \mathcal{T}_{h},\left.\nabla \cdot \boldsymbol{u}_{h}\right|_{K} \in \mathbb{R}$ and $\left.\boldsymbol{v}_{b}\right|_{\partial K}=\mathbf{0}$, the second equation in 2.5 corresponds to the weak form of the following problem

$$
\begin{aligned}
-\nu \Delta \boldsymbol{u}_{e}+\nabla p & =\boldsymbol{f}+\nu \Delta \boldsymbol{u}_{h} \quad \text { in } K \in \mathcal{T}_{h}, \\
\boldsymbol{u}_{e} & =\boldsymbol{g}_{e} \quad \text { on } F \subset \partial K \in \mathcal{T}_{h},
\end{aligned}
$$

where $\boldsymbol{g}_{e}$ is the solution of the following one-dimensional Poisson problem on $\mathcal{E}_{h}^{\text {int }}$ :

$$
\begin{aligned}
-\nu \partial_{s s} \boldsymbol{g}_{e} & =\frac{1}{h_{F}} \llbracket \nu \partial_{\boldsymbol{n}} \boldsymbol{u}_{h}+p \mathbf{I} \cdot \boldsymbol{n} \rrbracket_{F} \quad \text { on } F \in \mathcal{E}_{h}^{\text {int }}, \\
\boldsymbol{g}_{e} & =\mathbf{0} \quad \text { at the endpoints of } F .
\end{aligned}
$$

Here $s$ is the curvilinear abscissa of $F$, while $\llbracket \boldsymbol{w} \rrbracket_{F}$ denotes the jump of $\boldsymbol{w} \in \boldsymbol{H}^{1}(\Omega)$ across the edge $F$, that is

$$
\llbracket \boldsymbol{w} \rrbracket_{F}=\left.\left(\left.\boldsymbol{w}\right|_{K_{1}}\right)\right|_{F} \cdot \boldsymbol{n}_{1}+\left.\left(\left.\boldsymbol{w}\right|_{K_{2}}\right)\right|_{F} \cdot \boldsymbol{n}_{2},
$$

where $K_{1}, K_{2} \in \mathcal{T}_{h}$ are such that $K_{1} \cap K_{2}=F$ and $\boldsymbol{n}_{1}, \boldsymbol{n}_{2}$ are the exterior normals to $K_{1}, K_{2}$ respectively (see Figure 1). If $F$ lies on $\partial \Omega$, then we take $\llbracket \boldsymbol{w} \rrbracket_{F}=\boldsymbol{w} \cdot \boldsymbol{n}$. Note that the conformity of the enriched space for the bubble-part of the velocity is achieved via the non-homogeneous transmission condition on $\mathcal{E}_{h}^{\text {int }}$ defined by (2.6)-2.7). Now, on each $K \in \mathcal{T}_{h}$ set $\left.\boldsymbol{u}_{e}\right|_{K}=\boldsymbol{u}_{e}^{K}+\boldsymbol{u}_{e}^{\partial K}$. Therefore, from 2.6. we have the auxiliary problems:

$$
\begin{aligned}
-\nu \Delta \boldsymbol{u}_{e}^{K} & =\boldsymbol{f}+\nu \Delta \boldsymbol{u}_{h}-\nabla p \quad \text { in } K \in \mathcal{T}_{h}, \\
\boldsymbol{u}_{e}^{K} & =\mathbf{0} \quad \text { on } \partial K \in \mathcal{T}_{h},
\end{aligned}
$$

and

$$
\begin{aligned}
-\nu \Delta \boldsymbol{u}_{e}^{\partial K} & =0 \quad \text { in } K \in \mathcal{T}_{h}, \\
\boldsymbol{u}_{e}^{\partial K} & =\boldsymbol{g}_{e} \quad \text { on } \partial K \in \mathcal{T}_{h} .
\end{aligned}
$$


These problems are wellposed, and this implies that the second equation in (2.5) is satisfied. Then the enriched part of the solution is completely identified. A static condensation procedure (see the detailed development in [2]) allows to derive the following stabilized method: Find $\left(\boldsymbol{u}_{h}, p_{h}\right) \in \mathcal{V}_{h} \times Q_{h}$ such that

$$
\begin{gathered}
\nu\left(\nabla \boldsymbol{u}_{h}, \nabla \boldsymbol{v}_{h}\right)_{\Omega}-\left(p_{h}, \nabla \cdot \boldsymbol{v}_{h}\right)_{\Omega}+\left(q_{h}, \nabla \cdot \boldsymbol{u}_{h}\right)_{\Omega}+\sum_{K \in \mathcal{T}_{h}} \frac{h_{K}^{2}}{8 \nu}\left(-\nu \Delta \boldsymbol{u}_{h}+\nabla p_{h}, \nu \Delta \boldsymbol{v}_{h}+\nabla q_{h}\right)_{K} \\
\quad+\sum_{F \in \mathcal{E}_{h}^{\text {int }}} \frac{h_{F}}{12 \nu}\left(\llbracket \nu \partial_{\boldsymbol{n}} \boldsymbol{u}_{h} \rrbracket_{F}, \llbracket \nu \partial_{\boldsymbol{n}} \boldsymbol{v}_{h} \rrbracket_{F}\right)_{F}=\left(\boldsymbol{f}, \boldsymbol{v}_{h}\right)_{\Omega}+\sum_{K \in \mathcal{T}_{h}} \frac{h_{K}^{2}}{8 \nu}\left(\boldsymbol{f}, \nu \Delta \boldsymbol{v}_{h}+\nabla q_{h}\right)_{K},
\end{gathered}
$$

for all $\left(\boldsymbol{v}_{h}, q_{h}\right) \in \mathcal{V}_{h} \times Q_{h}$. Such formulation depends on the assumption that $\boldsymbol{f}$ is piecewise constant on each element $K \in \mathcal{T}_{h}$. Nevertheless, as done in [2], error estimates with the same optimal order of convergence can be derived for the more general case in which $\boldsymbol{f} \in \boldsymbol{H}^{1}(\Omega)$. Notice that in the case in which the jump terms are neglected, the method (2.9) reduces to a Douglas-Wang stabilization method (see e.g. [27]).

The following section contains well known results that will play a key role in the construction of the error estimates.

2.3. Some Technical Lemmas. We will make use of two well established trace inequalities (cf. [1, Th. $3.10])$

$$
\begin{aligned}
\|\boldsymbol{v}\|_{\boldsymbol{L}^{2}(F)}^{2} \leq C\left(h_{K}^{-1}\|\boldsymbol{v}\|_{\boldsymbol{L}^{2}(K)}^{2}+h_{K}|\boldsymbol{v}|_{\boldsymbol{H}^{1}(K)}^{2}\right) & \forall \boldsymbol{v} \in \boldsymbol{H}^{1}(K), \\
\left\|\partial_{\boldsymbol{n}} \boldsymbol{v}\right\|_{\boldsymbol{L}^{2}(F)}^{2} \leq C\left(h_{K}^{-1}|\boldsymbol{v}|_{\boldsymbol{H}^{1}(K)}^{2}+h_{K}|\boldsymbol{v}|_{\boldsymbol{H}^{2}(K)}^{2}\right) & \forall \boldsymbol{v} \in \boldsymbol{H}^{2}(K),
\end{aligned}
$$

for $F \in \partial K$, where $C$ depends also on the minimum angle of $K \in \mathcal{T}_{h}$.

Let $I_{h}: \boldsymbol{H}_{0}^{1}(\Omega) \cap C^{0}(\bar{\Omega})^{2} \rightarrow \mathcal{V}_{h}$ be the usual Lagrange interpolation operator, $\Pi_{h}: L^{2}(\Omega) \rightarrow Q_{h}$ the $L^{2}$-projection operator, and $J_{h}: \boldsymbol{H}^{1}(\Omega) \rightarrow \mathcal{V}_{h}$ the Clément interpolation operator (see e.g. [9, 13]). These operators satisfy some well known approximation properties which we collect in the following lemma.

Lemma 2.1 (Interpolation operators). For all $\boldsymbol{v} \in \boldsymbol{H}^{2}(\Omega), q \in H^{1}(\Omega) \cap L_{0}^{2}(\Omega), K \in \mathcal{T}_{h}, F \in \mathcal{E}_{h}^{\text {int }}$, there holds

$$
\begin{aligned}
\left|\boldsymbol{v}-I_{h} \boldsymbol{v}\right|_{\boldsymbol{H}^{m}(K)} \leq C h_{K}^{2-m}|\boldsymbol{v}|_{\boldsymbol{H}^{2}(K)} & m=0,1,2 \\
\left\|I_{h} \boldsymbol{v}\right\|_{\boldsymbol{H}^{1}(\Omega)} \leq C\|\boldsymbol{v}\|_{\boldsymbol{H}^{1}(\Omega)}, & \\
\left|\boldsymbol{v}-I_{h} \boldsymbol{v}\right|_{\boldsymbol{H}^{m}(F)} \leq C h_{F}^{2-m-1 / 2}|\boldsymbol{v}|_{\boldsymbol{H}^{2}(\tilde{K})} & m=0,1, \\
\left|\boldsymbol{v}-J_{h} \boldsymbol{v}\right|_{\boldsymbol{H}^{m}(K)} \leq C h_{K}^{1-m}|\boldsymbol{v}|_{\boldsymbol{H}^{1}(\tilde{K})} & m=0,1, \\
\left\|q-\Pi_{h} q\right\|_{L^{2}(\Omega)} \leq C h|q|_{H^{1}(\Omega)}, & \\
\left\|\Pi_{h} q\right\|_{L^{2}(\Omega)} \leq C\|q\|_{L^{2}(\Omega)}, &
\end{aligned}
$$

where $\tilde{K}$ is the union of all elements $L$ such that $\bar{K} \cap \bar{L} \neq \emptyset$.

Proof. For 2.12), 2.13), and (2.15)-(2.17) see e.g. 13, 26]. Relation (2.14) follows from (2.12), the local mesh regularity condition (that is, for $F \in \partial K$, there exists $C>0$ such that $\left.h_{F} \leq h_{K} \leq C h_{F}\right)$, and (2.10).

Owing to the continuous inf-sup condition satisfied by (2.4), it is known (cf. [18) that the following result holds.

Lemma 2.2. For each $r_{h} \in Q_{h} \subset L_{0}^{2}(\Omega)$, there exists $\boldsymbol{w} \in \boldsymbol{H}_{0}^{1}(\Omega)$ such that

$$
\nabla \cdot \boldsymbol{w}=r_{h} \text { a.e. in } \Omega \text {, and }|\boldsymbol{w}|_{\boldsymbol{H}^{1}(\Omega)} \leq C\left\|r_{h}\right\|_{L^{2}(\Omega)} .
$$

Finally, we recall the following regularity result for the dual problem (see [18]). 


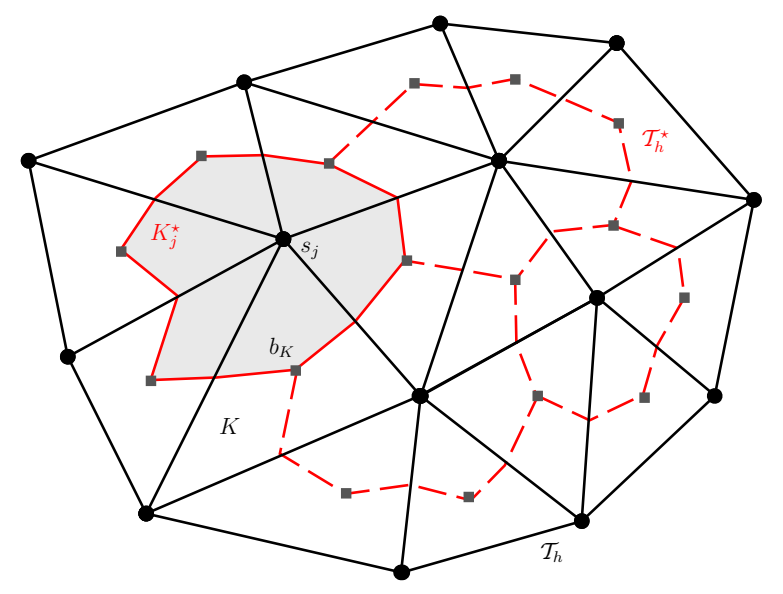

FiguRE 2. Schematic representation of elements in the primal mesh $\mathcal{T}_{h}$ and interior nodecentered control volumes of the dual mesh $\mathcal{T}_{h}^{\star}$ (in dashed lines).

Lemma 2.3. For any given $\boldsymbol{\varphi} \in \boldsymbol{L}^{2}(\Omega)$, consider the following dual problem: Find $(\boldsymbol{z}, s) \in\left[\boldsymbol{H}^{2}(\Omega) \cap\right.$ $\left.\boldsymbol{H}_{0}^{1}(\Omega)\right] \times\left[H^{1}(\Omega) \cap L_{0}^{2}(\Omega)\right]$ such that

$$
\begin{aligned}
-\nu \Delta \boldsymbol{z}-\nabla s=\boldsymbol{\varphi} & \text { in } \Omega, \\
\nabla \cdot \boldsymbol{z}=0 & \text { in } \Omega, \\
\boldsymbol{z}=\mathbf{0} & \text { on } \partial \Omega,
\end{aligned}
$$

and its weak form

$$
\nu(\nabla \boldsymbol{z}, \nabla \boldsymbol{v})_{\Omega}+(s, \nabla \cdot \boldsymbol{v})_{\Omega}-(q, \nabla \cdot \boldsymbol{z})_{\Omega}=(\boldsymbol{v}, \boldsymbol{\varphi})_{\Omega} \quad \forall(\boldsymbol{v}, q) \in \boldsymbol{H}_{0}^{1}(\Omega) \times L_{0}^{2}(\Omega) .
$$

If $\Omega$ is convex with Lipschitz-continuous boundary, then for the solution pair $(\boldsymbol{z}, s)$ the following well known estimate holds

$$
\|\boldsymbol{z}\|_{\boldsymbol{H}^{2}(\Omega)}+\|s\|_{H^{1}(\Omega)} \leq C\|\boldsymbol{\varphi}\|_{\boldsymbol{L}^{2}(\Omega)} .
$$

\section{Finite Volume Approximation}

In this section, starting from the FE method (2.9), and a standard finite volume mesh, we provide the main tools that stand behind our FVE formulation.

3.1. The finite volume mesh. Let $S=\left\{s_{j}, j=1, \ldots, N_{h}\right\}$ be the set of nodes of $\mathcal{T}_{h}$. Before defining our FVE method, let us introduce a dual mesh $\mathcal{T}_{h}^{\star}$ in $\Omega$, whose elements $K_{j}^{\star}$ are called control volumes. For constructing $\mathcal{T}_{h}^{\star}$, a general scheme for a generic triangle will be presented, however an analogous construction can be carried out if $\mathcal{T}_{h}$ is made of tetrahedra. If we fix an interior point $b_{K}$ in every $K \in \mathcal{T}_{h}$ (we will choose $b_{K}$ to be the barycenter of $K \in \mathcal{T}_{h}$ ), we can construct $\mathcal{T}_{h}^{\star}$ by associating to each node $s_{j} \in S$, a control volume $K_{j}^{\star}$, whose edges are obtained by connecting $b_{K}$ with the midpoints of each edge of $K$, forming a so-called Donald diagram (see e.g. [26]), as shown in Figure 2. If $\mathcal{T}_{h}$ is locally regular then so is $\mathcal{T}_{h}^{\star}$ (i.e., there exists $C>0$ such that $C^{-1} h^{2} \leq\left|K_{j}^{\star}\right| \leq C h^{2}$, for all $\left.K_{j}^{\star} \in \mathcal{T}_{h}^{\star}\right)$. In our FVE scheme, the trial function space for the velocity field associated with $\mathcal{T}_{h}$ is $\mathcal{V}_{h}$, and the test function space associated with $\mathcal{T}_{h}^{\star}$ corresponds to the set of all piecewise constants. Specifically,

$$
\mathcal{V}_{h}^{\star}:=\left\{\boldsymbol{v} \in \boldsymbol{L}^{2}(\Omega):\left.\boldsymbol{v}\right|_{K_{j}^{\star}} \in \mathbb{P}_{0}\left(K_{j}^{\star}\right)^{2} \text { for all } K_{j}^{\star} \in \mathcal{T}_{h}^{\star},\left.\boldsymbol{v}\right|_{K_{j}^{\star}}=\mathbf{0} \text { if } K_{j}^{\star} \text { is a boundary volume }\right\} .
$$

It holds that $\operatorname{dim}\left(\mathcal{V}_{h}\right)=\operatorname{dim}\left(\mathcal{V}_{h}^{\star}\right)=N_{h}$. Analogously, by $Q_{h}^{0}$ we denote the space of piecewise constant functions on every control volume $K_{j}^{\star}$, which is the test space for the pressure field associated with $\mathcal{T}_{h}^{\star}$. 
The relation between the trial and test spaces is made precise by the map $\mathcal{P}_{h}: \mathcal{V}_{h} \rightarrow \mathcal{V}_{h}^{\star}(\mathrm{cf}$. 3]) which is defined as follows: For all $\boldsymbol{v}_{h} \in \mathcal{V}_{h}$,

$$
\boldsymbol{v}_{h}(x)=\sum_{j=1}^{N_{h}} \boldsymbol{v}_{h}\left(s_{j}\right) \phi_{j}(x) \mapsto \mathcal{P}_{h} \boldsymbol{v}_{h}(x)=\sum_{j=1}^{N_{h}} \boldsymbol{v}_{h}\left(s_{j}\right) \chi_{j}(x) \quad x \in \Omega,
$$

where $\chi_{j}$ is the characteristic function of the control volume $K_{j}^{\star}$, that is,

$$
\chi_{j}(x)= \begin{cases}1 & x \in K_{j}^{\star} \\ 0 & \text { otherwise. }\end{cases}
$$

Note that $\left\{\boldsymbol{\chi}_{j}\right\}_{j}$ provides a basis of the finite volume space $\mathcal{V}_{h}^{\star}$. Note also that the map $\mathcal{P}_{h}$ is invertible. The operator $\mathcal{P}_{h}$ allows us to recast the Petrov-Galerkin formulation as a standard Galerkin method. The following lemma (cf. [10, 28] ) establishes a technical result involving the previously defined transfer operator.

Lemma 3.1. Let $K \in \mathcal{T}_{h}, F \subset \partial K$. Then there holds

$$
\begin{aligned}
\int_{K}\left(\boldsymbol{v}_{h}-\mathcal{P}_{h} \boldsymbol{v}_{h}\right) & =0 \\
\left\|\boldsymbol{v}_{h}-\mathcal{P}_{h} \boldsymbol{v}_{h}\right\|_{L^{2}(K)} & \leq C h_{K}\left|\boldsymbol{v}_{h}\right|_{\boldsymbol{H}^{1}(K)}, \\
\left\|\llbracket \partial_{\boldsymbol{n}} \mathcal{P}_{h} \boldsymbol{v}_{h} \rrbracket_{F}\right\|_{L^{2}(F)} & \leq\left\|\llbracket \partial_{\boldsymbol{n}} \boldsymbol{v}_{h} \rrbracket_{F}\right\|_{L^{2}(F)},
\end{aligned}
$$

for all $\boldsymbol{v}_{h} \in \mathcal{V}_{h} \cap \boldsymbol{H}^{1}(\Omega)$.

Moreover, if $\llbracket \partial_{\boldsymbol{n}} \boldsymbol{v}_{h} \rrbracket_{F}=0$ then $\llbracket \partial_{\boldsymbol{n}} \mathcal{P}_{h} \boldsymbol{v}_{h} \rrbracket_{F}=0$ (see $\left[10\right.$ ). Now, let $\boldsymbol{w} \in \mathcal{V}_{h} \cap \boldsymbol{H}^{2}(\Omega)$ and $F \in \mathcal{E}_{h}^{\text {int }}$. Using (3.3), the jump definition 2.8, the regularity of the mesh, and the trace inequality 2.11, we can deduce that

$$
\begin{aligned}
\sum_{F \in \mathcal{E}_{h}^{\text {int }}} h_{F}\left\|\llbracket \partial_{\boldsymbol{n}} \mathcal{P}_{h} \boldsymbol{w} \rrbracket_{F}\right\|_{L^{2}(F)}^{2} & \leq C \sum_{F \in \mathcal{E}_{h}^{\text {int }}} h_{F}\left\|\llbracket \partial_{\boldsymbol{n}} \boldsymbol{w} \rrbracket_{F}\right\|_{L^{2}(F)}^{2} \\
& \leq C \sum_{F \in \mathcal{E}_{h}^{\text {int }}} h_{F} \int_{F}\left(\left.\partial_{\boldsymbol{n}} \boldsymbol{w}\right|_{F}\right)^{2} \\
& \leq C \sum_{K \in \mathcal{T}_{h}}\left(|\boldsymbol{w}|_{\boldsymbol{H}^{1}(K)}^{2}+h_{K}^{2}|\boldsymbol{w}|_{\boldsymbol{H}^{2}(K)}^{2}\right)
\end{aligned}
$$

In the forthcoming analysis the following mesh-dependent norms will be used:

$$
\|\boldsymbol{v}\|_{h}:=\left(\nu|\boldsymbol{v}|_{\boldsymbol{H}^{1}(\Omega)}^{2}+\sum_{F \in \mathcal{E}_{h}^{\text {int }}} \frac{h_{F}}{12 \nu}\left\|\llbracket \nu \partial_{\boldsymbol{n}} \mathcal{P}_{h} \boldsymbol{v} \rrbracket_{F}\right\|_{L^{2}(F)}^{2}\right)^{1 / 2}, \quad\|q\|_{h}:=\left(\sum_{K \in \mathcal{T}_{h}} \frac{h_{k}^{2}}{8 \nu}|q|_{H^{1}(K)}^{2}\right)^{1 / 2} .
$$

3.2. Construction of the Stabilized FVE Method. Let $\left(\boldsymbol{v}_{h}, q_{h}\right) \in \mathcal{V}_{h} \times Q_{h}$. In order to construct the underlying FVE method, we consider the discrete problem associated to the variational formulation obtained by multiplying (2.1) by $\mathcal{P}_{h} \boldsymbol{v}_{h}$ and integrating by parts over each control volume $K_{j}^{\star} \in \mathcal{T}_{h}^{\star}$, then by multiplying (2.2) by $q_{h}$ and integrating by parts over each element $K \in \mathcal{T}_{h}$. We end up with the following finite volume element method: Find $\left(\boldsymbol{w}_{h}, r_{h}\right) \in \mathcal{V}_{h} \times Q_{h}$ such that

$$
\tilde{a}\left(\boldsymbol{w}_{h}, \mathcal{P}_{h} \boldsymbol{v}_{h}\right)+\tilde{b}\left(r_{h}, \mathcal{P}_{h} \boldsymbol{v}_{h}\right)+\left(q_{h}, \nabla \cdot \boldsymbol{w}_{h}\right)_{\Omega}=\left(\boldsymbol{f}, \mathcal{P}_{h} \boldsymbol{v}_{h}\right)_{\Omega} \quad \forall\left(\boldsymbol{v}_{h}, q_{h}\right) \in \mathcal{V}_{h} \times Q_{h},
$$

where the bilinear forms $\tilde{a}(\cdot, \cdot), \tilde{b}(\cdot, \cdot)$ are defined as follows:

$$
\tilde{a}\left(\boldsymbol{w}_{h}, \mathcal{P}_{h} \boldsymbol{v}_{h}\right)=-\sum_{j=1}^{N_{h}} \boldsymbol{v}_{h}\left(s_{j}\right) \int_{\partial K_{j}^{\star}} \nu \partial_{\boldsymbol{n}} \boldsymbol{w}_{h}, \quad \tilde{b}\left(r_{h}, \mathcal{P}_{h} \boldsymbol{v}_{h}\right)=\sum_{j=1}^{N_{h}} \boldsymbol{v}_{h}\left(s_{j}\right) \int_{\partial K_{j}^{\star}} r_{h} \boldsymbol{n},
$$

for $\boldsymbol{w}_{h}, \boldsymbol{v}_{h} \in \mathcal{V}_{h}, q_{h}, r_{h} \in Q_{h}$. A stabilized version of 3.5 will be introduced later. Notice that since the test 


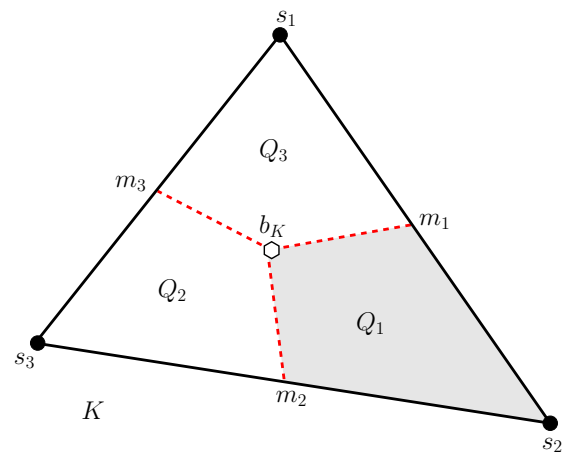

Figure 3. A given element $K$ of the primal mesh $\mathcal{T}_{h}$. The $m_{i}$ 's are the midpoints of the edges, $b_{K}$ is the barycenter of $K$ and the $Q_{i}$ 's are the quadrilaterals formed by the paths $b_{K} m_{i} s_{i+1} m_{i+1} b_{K}$.

functions are piecewise constant, the bilinear forms do not involve area integral terms as usually happens in FE formulations of Stokes problems.

Concerning these bilinear forms, the following result will be useful to carry out the error analysis in a finite-element-fashion (see e.g. 30]).

Lemma 3.2. For the bilinear forms $\tilde{a}(\cdot, \cdot), \tilde{b}(\cdot, \cdot)$ there holds:

$$
\begin{array}{lc}
\tilde{a}\left(\boldsymbol{w}_{h}, \mathcal{P}_{h} \boldsymbol{v}_{h}\right)=\nu\left(\nabla \boldsymbol{w}_{h}, \nabla \boldsymbol{v}_{h}\right)_{\Omega} \quad \forall \boldsymbol{w}_{h}, \boldsymbol{v}_{h} \in \mathcal{V}_{h}, \\
\tilde{b}\left(q_{h}, \mathcal{P}_{h} \boldsymbol{v}_{h}\right)=-\left(q_{h}, \nabla \cdot \boldsymbol{v}_{h}\right)_{\Omega} \quad \forall\left(\boldsymbol{v}_{h}, q_{h}\right) \in \mathcal{V}_{h} \times Q_{h} .
\end{array}
$$

Proof. First, let $g$ be a continuous function in the interior of a quadrilateral $Q_{j}$ (as shown in Figure 3 such that $\int_{F} g=0$ for every edge $F$ of $Q_{j}$. With the help of Figure 3 it is not hard to see that the following relation holds

$$
\sum_{j=1}^{N_{h}} \int_{\partial K_{j}^{*}} g=\sum_{K \in \mathcal{T}_{h}} \sum_{i=1}^{3} \int_{m_{i+1} b_{K} m_{i}} g,
$$

where $m_{i+1} b_{K} m_{i}$ stands for the union of the segments $m_{i+1} b_{K}$ and $b_{K} m_{i}$. In the case that the index is out of bound, we take $m_{i+1}=m_{i}$.

Next, any $\boldsymbol{v}_{h} \in \mathcal{V}_{h}$ is linear on each $\overline{a b} \subset F \in \mathcal{E}_{h}^{\text {int }}$. Then, in particular $\int_{a b} \boldsymbol{v}_{h}=1 / 2(a-b)\left(\boldsymbol{v}_{h}(a)+\boldsymbol{v}_{h}(b)\right)$ which implies that

$$
\int_{s_{j} s_{j+1}} \boldsymbol{v}_{h}=\int_{s_{j} m_{j}} \boldsymbol{v}_{h}\left(s_{j}\right)+\int_{m_{j} s_{j+1}} \boldsymbol{v}_{h}\left(s_{j+1}\right),
$$

where $s_{j}$ is a node of $\mathcal{T}_{h}$ and $m_{j}$ is the midpoint on the edge joining $s_{j}$ and $s_{j+1}$ (if $j=3$, then we take $\left.s_{j+1}=s_{1}\right)$.

Now, for obtaining (3.6) we use the definition of $\tilde{a}(\cdot, \cdot),(3.8)$, the fact that $\boldsymbol{v}_{h}\left(s_{i}\right)$ is constant in $Q_{i}$, and integration by parts twice to get

$$
\begin{aligned}
\tilde{a}\left(\boldsymbol{w}_{h}, \mathcal{P}_{h} \boldsymbol{v}_{h}\right) & =-\nu \sum_{K \in \mathcal{T}_{h}} \sum_{i=1}^{3} \boldsymbol{v}_{h}\left(s_{i}\right) \int_{m_{i+1} b_{K} m_{i}} \partial_{\boldsymbol{n}} \boldsymbol{w}_{h} \\
& =\nu \sum_{K \in \mathcal{T}_{h}} \sum_{i=1}^{3} \boldsymbol{v}_{h}\left(s_{i}\right) \int_{m_{i} s_{i+1} m_{i+1}} \partial_{\boldsymbol{n}} \boldsymbol{w}_{h}-\nu \sum_{K \in \mathcal{T}_{h}} \sum_{Q_{i}}\left(\Delta \boldsymbol{w}_{h}, \boldsymbol{v}_{h}\left(s_{i}\right)\right)_{Q_{i}}
\end{aligned}
$$


BUBBLE-LIKE STABILIZED FINITE VOLUME ELEMENT METHOD

$$
\begin{aligned}
& =\nu \sum_{K \in \mathcal{T}_{h}} \sum_{i=1}^{3} \boldsymbol{v}_{h}\left(s_{i}\right) \int_{m_{i} s_{i+1} m_{i+1}}\left(\boldsymbol{v}_{h}\left(s_{i}\right)-\boldsymbol{v}_{h}\right) \cdot \partial_{\boldsymbol{n}} \boldsymbol{w}_{h}+\nu\left(\nabla \boldsymbol{w}_{h}, \nabla \boldsymbol{v}_{h}\right)_{\Omega} \\
& =\nu \sum_{K \in \mathcal{T}_{h}} \sum_{i=1}^{3} \boldsymbol{v}_{h}\left(s_{i}\right)\left[\int_{s_{i} m_{i}} \boldsymbol{v}_{h}\left(s_{i}\right)+\int_{s_{i} m_{i+1}} \boldsymbol{v}_{h}\left(s_{i+1}\right)-\int_{s_{i} s_{i+1}} \boldsymbol{v}_{h}\right]+\nu\left(\nabla \boldsymbol{w}_{h}, \nabla \boldsymbol{v}_{h}\right)_{\Omega} .
\end{aligned}
$$

Noticing that $\partial_{\boldsymbol{n}} \boldsymbol{v}_{h}$ is constant on the edges of $K$, and after applying (3.9), we get (3.6). For proving (3.7), we use the definition of $\mathcal{P}_{h}$, integration by parts and (3.1) to obtain

$$
\begin{aligned}
\tilde{b}\left(q_{h}, \mathcal{P}_{h} \boldsymbol{v}_{h}\right) & =\sum_{j=1}^{N_{h}} \boldsymbol{v}_{h}\left(s_{j}\right) \int_{\partial K_{j}^{\star}} q_{h} \boldsymbol{n} \\
& =\sum_{j=1}^{N_{h}} \int_{K_{j}^{\star}} \mathcal{P}_{h} \boldsymbol{v}_{h} \nabla q_{h}=\sum_{K \in \mathcal{T}_{h}} \sum_{i=1}^{3} \int_{Q_{i}} \mathcal{P}_{h} \boldsymbol{v}_{h} \nabla q_{h} \\
& =\sum_{K \in \mathcal{T}_{h}} \int_{K}\left(\mathcal{P}_{h} \boldsymbol{v}_{h}-\boldsymbol{v}_{h}\right) \nabla q_{h}+\sum_{K \in \mathcal{T}_{h}} \int_{K} \boldsymbol{v}_{h} \nabla q_{h}=\sum_{K \in \mathcal{T}_{h}} \int_{K} \boldsymbol{v}_{h} \nabla q_{h} \\
& =-\left(q_{h}, \nabla \cdot \boldsymbol{v}_{h}\right)_{\Omega} .
\end{aligned}
$$

Corollary 3.1. The bilinear form $\tilde{a}(\cdot, \cdot)$ is symmetric, continuous and coercive in $\mathcal{V}_{h}$.

We point out that a similar analysis can be carried out if instead of considering dual meshes of Donaldtype, we use the so-called Voronoi-type (see e.g. [26]) dual meshes.

With our choice for the spaces $\mathcal{V}_{h} \times Q_{h}$ (i.e., a $\mathbb{P}_{1}-\mathbb{P}_{1}$ pair) the finite volume scheme (3.5) does not satisfy the discrete inf-sup condition. Therefore we incorporate the same stabilization terms showing up in the finite element formulation 2.9. This implies that the proposed stabilized FVE method reads: Find $\left(\tilde{\boldsymbol{u}}_{h}, \tilde{p}_{h}\right) \in \mathcal{V}_{h} \times Q_{h}$ such that

$$
\begin{aligned}
& \tilde{a}\left(\tilde{\boldsymbol{u}}_{h}, \mathcal{P}_{h} \boldsymbol{v}_{h}\right)+\tilde{b}\left(\tilde{p}_{h}, \mathcal{P}_{h} \boldsymbol{v}_{h}\right)+\left(q_{h}, \nabla \cdot \tilde{\boldsymbol{u}}_{h}\right)_{\Omega}+\sum_{K \in \mathcal{T}_{h}} \frac{h_{K}^{2}}{8 \nu}\left(-\nu \Delta \tilde{\boldsymbol{u}}_{h}+\nabla \tilde{p}_{h}, \nu \Delta \boldsymbol{v}_{h}+\nabla q_{h}\right)_{K} \\
& \quad+\sum_{F \in \mathcal{E}_{h}^{\text {int }}} \frac{h_{F}}{12 \nu}\left(\llbracket \nu \partial_{\boldsymbol{n}} \mathcal{P}_{h} \tilde{\boldsymbol{u}}_{h} \rrbracket_{F}, \llbracket \nu \partial_{\boldsymbol{n}} \mathcal{P}_{h} \boldsymbol{v}_{h} \rrbracket_{F}\right)_{F}=\left(\boldsymbol{f}, \mathcal{P}_{h} \boldsymbol{v}_{h}\right)_{\Omega}+\sum_{K \in \mathcal{T}_{h}} \frac{h_{K}^{2}}{8 \nu}\left(\boldsymbol{f}, \nu \Delta \boldsymbol{v}_{h}+\nabla q_{h}\right)_{K},
\end{aligned}
$$

for all $\left(\boldsymbol{v}_{h}, q_{h}\right) \in \mathcal{V}_{h} \times Q_{h}$. In the light of Lemma 3.2 , it can be recast as: Find $\left(\tilde{\boldsymbol{u}}_{h}, \tilde{p}_{h}\right) \in \mathcal{V}_{h} \times Q_{h}$ such that

$$
\mathcal{C}_{h}\left(\left(\tilde{\boldsymbol{u}}_{h}, \tilde{p}_{h}\right),\left(\boldsymbol{v}_{h}, q_{h}\right)\right)=\mathcal{F}_{h}\left(\boldsymbol{v}_{h}, q_{h}\right) \quad \forall\left(\boldsymbol{v}_{h}, q_{h}\right) \in \mathcal{V}_{h} \times Q_{h},
$$

where for all $\left(\boldsymbol{w}_{h}, p_{h}\right),\left(\boldsymbol{v}_{h}, q_{h}\right) \in \mathcal{V}_{h} \times Q_{h}$, the forms $\mathcal{C}_{h}$ and $\mathcal{F}_{h}$ are defined as follows

$$
\begin{aligned}
& \mathcal{C}_{h}\left(\left(\boldsymbol{w}_{h}, p_{h}\right),\left(\boldsymbol{v}_{h}, q_{h}\right)\right):=\nu\left(\nabla \boldsymbol{w}_{h}, \nabla \boldsymbol{v}_{h}\right)_{\Omega}-\left(p_{h}, \nabla \cdot \boldsymbol{v}_{h}\right)_{\Omega}+\left(q_{h}, \nabla \cdot \boldsymbol{w}_{h}\right)_{\Omega} \\
& \quad+\sum_{K \in \mathcal{T}_{h}} \frac{h_{K}^{2}}{8 \nu}\left(-\nu \Delta \boldsymbol{w}_{h}+\nabla p_{h}, \nu \Delta \boldsymbol{v}_{h}+\nabla q_{h}\right)_{K}+\sum_{F \in \mathcal{E}_{h}^{\text {int }}} \frac{h_{F}}{12 \nu}\left(\llbracket \nu \partial_{\boldsymbol{n}} \mathcal{P}_{h} \boldsymbol{w}_{h} \rrbracket_{F}, \llbracket \nu \partial_{\boldsymbol{n}} \mathcal{P}_{h} \boldsymbol{v}_{h} \rrbracket_{F}\right)_{F}, \\
& \mathcal{F}_{h}\left(\boldsymbol{v}_{h}, q_{h}\right):=\left(\boldsymbol{f}, \mathcal{P}_{h} \boldsymbol{v}_{h}\right)_{\Omega}+\sum_{K \in \mathcal{T}_{h}} \frac{h_{K}^{2}}{8 \nu}\left(\boldsymbol{f}, \nu \Delta \boldsymbol{v}_{h}+\nabla q_{h}\right)_{K} .
\end{aligned}
$$

\section{Convergence Analysis}

The goal of this section is to derive the error analysis for (3.10). We will proceed by obtaining optimal error estimates in the $h$-norms, and in the $L^{2}-$ norm. 
Remark 4.1. In the whole section, we will consider that the solution $(\boldsymbol{u}, p)$ of $(2.4)$ belongs to $\left[\boldsymbol{H}^{2}(\Omega) \cap\right.$ $\left.\boldsymbol{H}_{0}^{1}(\Omega)\right] \times\left[H^{1}(\Omega) \cap L_{0}^{2}(\Omega)\right]$. Such regularity holds either if $\Omega$ is convex, $\partial \Omega$ is Lipschitz-continuous, and if $\boldsymbol{f}$ fulfils certain orthogonality relations given e.g. in [4, Th. II.1].

Lemma 4.1 (Consistency). Let the pair $(\boldsymbol{u}, p)$ be the solution of 2.4 and let $\left(\tilde{\boldsymbol{u}}_{h}, \tilde{p}_{h}\right) \in \mathcal{V}_{h} \times Q_{h}$ be its approximation defined by the FVE method (3.10). Then, if $\boldsymbol{f}$ is piecewise constant with respect to the primal triangulation $\mathcal{T}_{h}$, there holds that

$$
\mathcal{C}_{h}\left(\left(\boldsymbol{u}-\tilde{\boldsymbol{u}}_{h}, p-\tilde{p}_{h}\right),\left(\boldsymbol{v}_{h}, q_{h}\right)\right)=0 \quad \forall\left(\boldsymbol{v}_{h}, q_{h}\right) \in \mathcal{V}_{h} \times Q_{h},
$$

that is, the FVE method (3.10) is fully consistent.

Proof. Remark 4.1 implies that $\llbracket \nu \partial_{\boldsymbol{n}} \boldsymbol{u} \rrbracket_{F}$ vanishes on every internal edge $F$ of the primal mesh. Then, using (3.11), 3.1) and (3.2), the result follows.

If $f$ is not piecewise constant, then we only obtain asymptotic consistency (see e.g. [13]). Moreover, the loss of consistency induced by considering $f$ being piecewise constant is smaller than the order of the method. In fact,

$$
\begin{aligned}
\mathcal{C}_{h}\left(\left(\boldsymbol{u}-\tilde{\boldsymbol{u}}_{h}, p-\tilde{p}_{h}\right),\left(\boldsymbol{v}_{h}, q_{h}\right)\right) & =\mathcal{C}_{h}\left((\boldsymbol{u}, p),\left(\boldsymbol{v}_{h}, q_{h}\right)\right)-\mathcal{F}_{h}\left(\boldsymbol{v}_{h}, q_{h}\right) \\
& =\left(\boldsymbol{f}, \boldsymbol{v}_{h}\right)_{\Omega}-\left(\boldsymbol{f}, \mathcal{P}_{h} \boldsymbol{v}_{h}\right)_{\Omega}+\sum_{F \in \mathcal{E}_{h}^{\text {int }}} \frac{h_{F}}{12 \nu}\left(\llbracket \nu \partial_{\boldsymbol{n}} \mathcal{P}_{h} \boldsymbol{u} \rrbracket_{F}, \llbracket \nu \partial_{\boldsymbol{n}} \mathcal{P}_{h} \boldsymbol{v}_{h} \rrbracket_{F}\right)_{F} \\
& =\sum_{K \in \mathcal{T}_{h}}\left(\boldsymbol{f}, \boldsymbol{v}_{h}-\mathcal{P}_{h} \boldsymbol{v}_{h}\right)_{K} \\
& =\sum_{K \in \mathcal{T}_{h}}\left(\boldsymbol{f}-\int_{K} \boldsymbol{f}, \boldsymbol{v}_{h}-\mathcal{P}_{h} \boldsymbol{v}_{h}\right)_{K} \\
& \leq C h^{2}\|\boldsymbol{f}\|_{\boldsymbol{L}^{2}(\Omega)}\left|\boldsymbol{v}_{h}\right|_{\boldsymbol{H}^{1}(\Omega)},
\end{aligned}
$$

for all $\boldsymbol{v}_{h} \in \mathcal{V}_{h} \cap \boldsymbol{H}^{2}(\Omega)$, by virtue of Cauchy-Schwarz inequality and Lemma 3.1.

Note that from the definition of $\mathcal{C}_{h}, \mathcal{V}_{h}$ and that of the $h$-norms, the following result holds, which implies the well-posedness of 3.10 .

Lemma 4.2 (Continuity and coercivity in the $h$-norms). Let $\left(\boldsymbol{w}_{h}, r_{h}\right) \in \mathcal{V}_{h} \times Q_{h}$. Then

$$
\begin{aligned}
\mathcal{C}_{h}\left(\left(\boldsymbol{w}_{h}, r_{h}\right),\left(\boldsymbol{v}_{h}, q_{h}\right)\right) & \leq\left(\left\|\boldsymbol{w}_{h}\right\|_{h}+\left\|r_{h}\right\|_{h}\right)\left(\left\|\boldsymbol{v}_{h}\right\|_{h}+\left\|q_{h}\right\|_{h}\right), \\
\mathcal{C}_{h}\left(\left(\boldsymbol{v}_{h}, q_{h}\right),\left(\boldsymbol{v}_{h}, q_{h}\right)\right) & =\left\|\boldsymbol{v}_{h}\right\|_{h}^{2}+\left\|q_{h}\right\|_{h}^{2},
\end{aligned}
$$

for all $\left(\boldsymbol{v}_{h}, q_{h}\right) \in \mathcal{V}_{h} \times Q_{h}$.

Since the Clément interpolate $J_{h} q$ of $q \in H^{1}(\Omega) \cap L_{0}^{2}(\Omega)$, does not necessarily belong to $L_{0}^{2}(\Omega)$, we will introduce the operator $L_{h}$ defined by $L_{h} q:=J_{h} q-|\Omega|^{-1} \int_{\Omega} J_{h} q$. This operator possesses the same interpolation properties (e.g. 2.15) as $J_{h}$.

Theorem 4.1 (An optimal-order error estimate in the $h$-norms). Let $\left(\tilde{\boldsymbol{u}}_{h}, \tilde{p}_{h}\right) \in \mathcal{V}_{h} \times Q_{h}$ be the unique solution of (3.10) and $(\boldsymbol{u}, p)$ the unique solution of (2.4). Then, under the assumption of $\boldsymbol{f}$ being piecewise constant, there exists $C>0$ such that

$$
\left\|\boldsymbol{u}-\tilde{\boldsymbol{u}}_{h}\right\|_{h}+\left\|p-\tilde{p}_{h}\right\|_{h} \leq C h\left(|\boldsymbol{u}|_{H^{2}(\Omega)}+|p|_{H^{1}(\Omega)}\right) .
$$

Proof. Let $\varepsilon=I_{h} \boldsymbol{u}-\boldsymbol{u}, \eta=L_{h} p-p$ be the individual errors between the exact solution and the projected solution, and let $\varepsilon_{h}=I_{h} \boldsymbol{u}-\tilde{\boldsymbol{u}}_{h}, \eta_{h}=L_{h} p-\tilde{p}_{h}$ denote the error between the FVE approximation and the projection of the exact solution. 
First, using (3.4) and 2.12 we have

$$
\begin{aligned}
\sum_{F \in \mathcal{E}_{h}^{\text {int }}} \frac{h_{F}}{12 \nu}\left\|\llbracket \nu \partial_{\boldsymbol{n}} \mathcal{P}_{h}\left(\boldsymbol{v}-I_{h} \boldsymbol{v}\right) \rrbracket_{F}\right\|_{L^{2}(F)}^{2} & \leq C \sum_{K \in \mathcal{T}_{h}}\left(\left|\boldsymbol{v}-I_{h} \boldsymbol{v}\right|_{\boldsymbol{H}^{1}(K)}^{2}+h_{K}^{2}\left|\boldsymbol{v}-I_{h} \boldsymbol{v}\right|_{\boldsymbol{H}^{2}(K)}^{2}\right) \\
& \leq C h^{2}|\boldsymbol{v}|_{\boldsymbol{H}^{2}(\Omega)}^{2},
\end{aligned}
$$

and using the definition of the $h-$ norm and Lemma 2.1, gives

$$
\left\|\boldsymbol{v}-I_{h} \boldsymbol{v}\right\|_{h}^{2} \leq C h^{2}|\boldsymbol{v}|_{H^{2}(\Omega)}^{2} .
$$

Furthermore, 2.15) and the definition of the $h$-norm also implies that

$$
\nu^{-1}\left\|q-L_{h} q\right\|_{L^{2}(\Omega)}^{2}+\left\|q-L_{h} q\right\|_{h}^{2} \leq C \nu^{-1} h^{2}|q|_{H^{1}(\Omega)}^{2} .
$$

Next, applying 4.1, Lemma 4.1 and integration by parts we get

$$
\begin{aligned}
\left\|\varepsilon_{h}\right\|_{h}^{2}+\left\|\eta_{h}\right\|_{h}^{2}= & \mathcal{C}_{h}\left(\left(\varepsilon_{h}, \eta_{h}\right),\left(\varepsilon_{h}, \eta_{h}\right)\right) \\
= & \mathcal{C}_{h}\left(\left(\boldsymbol{u}-\tilde{\boldsymbol{u}}_{h}, p-\tilde{p}_{h}\right),\left(\boldsymbol{v}_{h}, q_{h}\right)\right)+\mathcal{C}_{h}\left((\varepsilon, \eta),\left(\varepsilon_{h}, \eta_{h}\right)\right) \\
= & \nu\left(\nabla \varepsilon, \nabla \varepsilon_{h}\right)_{\Omega}-\left(\eta, \nabla \cdot \varepsilon_{h}\right)_{\Omega}-\left(\varepsilon, \nabla \eta_{h}\right)_{\Omega} \\
& \quad+\sum_{K \in \mathcal{T}_{h}} \frac{h_{K}^{2}}{8 \nu}\left(-\nu \Delta \varepsilon+\nabla \eta, \nabla \eta_{h}\right)_{K}+\sum_{F \in \mathcal{E}_{h}^{\text {int }}} \frac{h_{F}}{12 \nu}\left(\llbracket \nu \partial_{\boldsymbol{n}} \mathcal{P}_{h} \varepsilon \rrbracket_{F}, \llbracket \nu \partial_{\boldsymbol{n}} \mathcal{P}_{h} \varepsilon_{h} \rrbracket_{F}\right)_{F} .
\end{aligned}
$$

Now, (4.5), Cauchy-Schwarz inequality, the definition of $h$-norms, a repeated application of (4.3), and 2.12, 2.14, 2.15), enable us to write

$$
\begin{gathered}
\left\|\varepsilon_{h}\right\|_{h}^{2}+\left\|\eta_{h}\right\|_{h}^{2} \leq C\left(|\varepsilon|_{\boldsymbol{H}^{1}(\Omega)}^{2}+\|\varepsilon\|_{\boldsymbol{L}^{2}(\Omega)}^{2}+\|\eta\|_{L^{2}(\Omega)}^{2}+\sum_{F \in \mathcal{E}_{h}^{\text {int }}} \frac{h_{F}}{12 \nu}\left\|\llbracket \nu \partial_{\boldsymbol{n}} \mathcal{P}_{h} \varepsilon \rrbracket_{F}\right\|_{L^{2}(F)}^{2}\right. \\
\left.+\sum_{K \in \mathcal{T}_{h}}\left[\frac{8 \nu}{h_{K}^{2}}\|\varepsilon\|_{\boldsymbol{L}^{2}(K)}^{2}+\frac{h_{K}^{2}}{8 \nu}\left(\|\eta\|_{L^{2}(K)}^{2}+\|\Delta \varepsilon\|_{\boldsymbol{L}^{2}(K)}^{2}\right)\right]\right)^{1 / 2} \\
\quad \times\left(\left|\varepsilon_{h}\right|_{\boldsymbol{H}^{1}(\Omega)}^{2}+\sum_{K \in \mathcal{T}_{h}} \frac{h_{K}^{2}}{8 \nu}\left\|\eta_{h}\right\|_{H^{1}(K)}^{2}+\sum_{F \in \mathcal{E}_{h}^{\text {int }}} \frac{h_{F}}{12 \nu}\left\|\llbracket \nu \partial_{\boldsymbol{n}} \mathcal{P}_{h} \varepsilon_{h} \rrbracket_{F}\right\|_{L^{2}(F)}^{2}\right)^{1 / 2} \\
\leq C\left(\|\varepsilon\|_{h}^{2}+\|\varepsilon\|_{\boldsymbol{L}^{2}(\Omega)}^{2}+\sum_{K \in \mathcal{T}_{h}}\left[\frac{8 \nu}{h_{K}^{2}}\|\varepsilon\|_{\boldsymbol{L}^{2}(K)}^{2}+\frac{h_{K}^{2}}{8 \nu}\|\Delta \varepsilon\|_{\boldsymbol{L}^{2}(K)}^{2}\right]+\|\eta\|_{h}^{2}+\|\eta\|_{L^{2}(\Omega)}^{2}\right)^{1 / 2} \\
\times\left(\left\|\varepsilon_{h}\right\|_{h}^{2}+\left\|\eta_{h}\right\|_{h}^{2}\right)^{1 / 2} \\
\leq C\left(h^{2}|\boldsymbol{u}|_{\boldsymbol{H}^{2}(\Omega)}^{2}+h^{2}|p|_{H^{1}(\Omega)}^{2}+\sum_{K \in \mathcal{T}_{h}} 8 \nu\left(1+\nu^{2}\right) h_{K}^{2}|\boldsymbol{u}|_{\boldsymbol{H}^{1}(K)}^{2}\right)^{1 / 2}\left(\left\|\varepsilon_{h}\right\|_{h}^{2}+\left\|\eta_{h}\right\|_{h}^{2}\right)^{1 / 2},
\end{gathered}
$$

which implies the following:

$$
\left\|\varepsilon_{h}\right\|_{h}+\left\|\eta_{h}\right\|_{h} \leq C h\left(|\boldsymbol{u}|_{\boldsymbol{H}^{2}(\Omega)}^{2}+|p|_{H^{1}(\Omega)}^{2}\right)^{1 / 2} .
$$

Finally, in order to get the desired result, it is sufficient to apply triangular inequality and (4.3), (4.4).

Theorem 4.2 (An optimal-order $L^{2}$-error estimate for the pressure field). Assume that $\left(\tilde{\boldsymbol{u}}_{h}, \tilde{p}_{h}\right) \in \mathcal{V}_{h} \times Q_{h}$ and $(\boldsymbol{u}, p)$ are the unique solutions of (3.10) and (2.4), respectively. Then, there exists a positive constant $C>0$ such that

$$
\left\|p-\tilde{p}_{h}\right\|_{L^{2}(\Omega)} \leq C h\left(|\boldsymbol{u}|_{\boldsymbol{H}^{2}(\Omega)}+|p|_{H^{1}(\Omega)}\right) .
$$


Proof. Let be $\boldsymbol{w} \in \boldsymbol{H}_{0}^{1}(\Omega)$ such that $\nabla \cdot \boldsymbol{w}=p-\tilde{p}_{h}$, as stated in Lemma 2.2. Further, selecting $\left(\boldsymbol{v}_{h}, q_{h}\right)=$ $\left(J_{h} \boldsymbol{w}, 0\right) \in \mathcal{V}_{h} \times Q_{h}$ in Lemma 4.1 we have

$$
0=\nu\left(\nabla\left(\boldsymbol{u}-\tilde{\boldsymbol{u}}_{h}\right), \nabla J_{h} \boldsymbol{w}\right)_{\Omega}-\left(p-\tilde{p}_{h}, \nabla \cdot J_{h} \boldsymbol{w}\right)_{\Omega}+\sum_{F \in \mathcal{E}_{h}^{\text {int }}} \frac{h_{F}}{12 \nu}\left(\llbracket \nu \partial_{\boldsymbol{n}} \mathcal{P}_{h}\left(\boldsymbol{u}-\tilde{\boldsymbol{u}}_{h}\right) \rrbracket_{F}, \llbracket \nu \partial_{\boldsymbol{n}} \mathcal{P}_{h} J_{h} \boldsymbol{w} \rrbracket_{F}\right)_{F} .
$$

Using this relation and integration by parts we obtain

$$
\begin{aligned}
\left\|p-\tilde{p}_{h}\right\|_{L^{2}(\Omega)}^{2}= & \left(p-\tilde{p}_{h}, \nabla \cdot \boldsymbol{w}\right)_{\Omega} \\
= & \left(p-\tilde{p}_{h}, \nabla \cdot\left(\boldsymbol{w}-J_{h} \boldsymbol{w}\right)\right)_{\Omega}+\left(p-\tilde{p}_{h}, \nabla \cdot J_{h} \boldsymbol{w}\right)_{\Omega} \\
= & -\sum_{K \in \mathcal{T}_{h}}\left(\boldsymbol{w}-J_{h} \boldsymbol{w}, \nabla\left(\left(p-\tilde{p}_{h}\right)\right)_{K}+\nu\left(\nabla\left(\boldsymbol{u}-\tilde{\boldsymbol{u}}_{h}\right), \nabla J_{h} \boldsymbol{w}\right)_{\Omega}\right. \\
& \quad+\sum_{F \in \mathcal{E}_{h}^{\text {int }}} \frac{h_{F}}{12 \nu}\left(\llbracket \nu \partial_{\boldsymbol{n}} \mathcal{P}_{h}\left(\boldsymbol{u}-\tilde{\boldsymbol{u}}_{h}\right) \rrbracket_{F}, \llbracket \nu \partial_{\boldsymbol{n}} \mathcal{P}_{h} J_{h} \boldsymbol{w} \rrbracket_{F}\right)_{F} .
\end{aligned}
$$

Then, by Cauchy-Schwarz inequality, 2.15, Lemma 2.2 2.10, definition of $h$-norms, and Theorem 4.1 we can infer that

$$
\begin{aligned}
\left\|p-\tilde{p}_{h}\right\|_{L^{2}(\Omega)}^{2} \leq & \sum_{K \in \mathcal{T}_{h}}\left\|\boldsymbol{w}-J_{h} \boldsymbol{w}\right\|_{\boldsymbol{L}^{2}(K)}\left|p-\tilde{p}_{h}\right|_{H^{1}(K)}+\nu\left|\boldsymbol{u}-\tilde{\boldsymbol{u}}_{h}\right|_{\boldsymbol{H}^{1}(\Omega)}\left|J_{h} \boldsymbol{w}\right|_{\boldsymbol{H}^{1}(\Omega)} \\
& +\sum_{F \in \mathcal{E}_{h}^{\text {int }}} \frac{h_{F}}{12 \nu}\left(\llbracket \nu \partial_{\boldsymbol{n}} \mathcal{P}_{h}\left(\boldsymbol{u}-\tilde{\boldsymbol{u}}_{h}\right) \rrbracket_{F}, \llbracket \nu \partial_{\boldsymbol{n}} \mathcal{P}_{h} J_{h} \boldsymbol{w} \rrbracket_{F}\right)_{F} \\
\leq & \left(\sum_{K \in \mathcal{T}_{h}} \frac{8 \nu}{h_{K}^{2}}\left\|\boldsymbol{w}-J_{h} \boldsymbol{w}\right\|_{\boldsymbol{L}^{2}(K)}^{2}+\nu\left|J_{h} \boldsymbol{w}\right|_{\boldsymbol{H}^{1}(\Omega)}+\sum_{F \in \mathcal{E}_{h}^{\text {int }}} \frac{h_{F}}{12 \nu}\left\|\llbracket \nu \partial_{\boldsymbol{n}} \mathcal{P}_{h} J_{h} \boldsymbol{w} \rrbracket_{F}\right\|_{\boldsymbol{L}^{2}(F)}^{2}\right)^{1 / 2} \\
& \times\left(\sum_{K \in \mathcal{T}_{h}} \frac{h_{K}^{2}}{8 \nu}\left|p-\tilde{p}_{h}\right|_{\boldsymbol{H}^{1}(K)}^{2}+\left|\boldsymbol{u}-\tilde{\boldsymbol{u}}_{h}\right|_{\boldsymbol{H}^{1}(\Omega)}^{2}+\sum_{F \in \mathcal{E}_{h}^{\text {int }}} \frac{h_{F}}{12 \nu}\left\|\llbracket \nu \partial_{\boldsymbol{n}} \mathcal{P}_{h}\left(\boldsymbol{u}-\tilde{\boldsymbol{u}}_{h}\right) \rrbracket_{F}\right\|_{\boldsymbol{L}^{2}(F)}^{2}\right)^{1 / 2} \\
\leq & C\left(|\boldsymbol{w}|_{\boldsymbol{H}^{1}(\Omega)}^{2}+\nu\left|J_{h} \boldsymbol{w}\right|_{\boldsymbol{H}^{1}(\Omega)}^{2}\right)^{1 / 2}\left(\left\|\boldsymbol{u}-\tilde{\boldsymbol{u}}_{h}\right\|_{h}^{2}+\left\|p-\tilde{p}_{h}\right\|_{h}^{2}\right)^{1 / 2} \\
\leq & C h\left\|p-\tilde{p}_{h}\right\|_{L^{2}(\Omega)}\left(|\boldsymbol{u}|_{\boldsymbol{H}^{2}(\Omega)}+|p|_{H^{1}(\Omega)}\right),
\end{aligned}
$$

and dividing by $\left\|p-\tilde{p}_{h}\right\|_{L^{2}(\Omega)}$, the result follows.

Theorem 4.3 ( $L^{2}$-error estimate for the velocity field). Suppose that $(\boldsymbol{u}, p)$ is the solution of (2.4), and $\left(\tilde{\boldsymbol{u}}_{h}, \tilde{p}_{h}\right) \in \mathcal{V}_{h} \times Q_{h}$ is the approximation defined by the FVE method $(3.10)$. Then the following a priori error estimate holds

$$
\left\|\boldsymbol{u}-\tilde{\boldsymbol{u}}_{h}\right\|_{\boldsymbol{L}^{2}(\Omega)} \leq C h^{2}\left(|\boldsymbol{u}|_{\boldsymbol{H}^{2}(\Omega)}+|p|_{H^{1}(\Omega)}\right) .
$$

Proof. First consider the dual problem (2.18) with $\boldsymbol{\varphi}=\boldsymbol{u}-\tilde{\boldsymbol{u}}_{h}$. Moreover, let us choose in (2.4) and (3.10), $\left(\boldsymbol{v}_{h}, q_{h}\right)=\left(I_{h} \boldsymbol{z}, \Pi_{h} s\right) \in \mathcal{V}_{h} \times Q_{h}$, and subtract the resulting expressions. We then subtract again the result to 2.18 with the particular choice $(\boldsymbol{v}, q)=\left(\boldsymbol{u}-\tilde{\boldsymbol{u}}_{h}, p-\tilde{p}_{h}\right)$, (and again $\boldsymbol{\varphi}=\boldsymbol{u}-\tilde{\boldsymbol{u}}_{h}$ ). Next we apply Lemma 4.1 to obtain

$$
\begin{aligned}
\left\|\boldsymbol{u}-\tilde{\boldsymbol{u}}_{h}\right\|_{\boldsymbol{L}^{2}(\Omega)}^{2}=\sum_{F \in \mathcal{E}_{h}^{\text {int }}} & \frac{h_{F}}{12 \nu}\left(\llbracket \nu \partial_{\boldsymbol{n}} \mathcal{P}_{h}\left(\boldsymbol{u}-\tilde{\boldsymbol{u}}_{h}\right) \rrbracket_{F}, \llbracket \nu \partial_{\boldsymbol{n}} \mathcal{P}_{h}\left(\boldsymbol{z}-I_{h} \boldsymbol{z}\right) \rrbracket_{F}-\llbracket \nu \partial_{\boldsymbol{n}} \mathcal{P}_{h} \boldsymbol{z} \rrbracket_{F}\right)_{F} \\
& +\nu\left(\nabla\left(\boldsymbol{u}-\tilde{\boldsymbol{u}}_{h}\right), \nabla\left(\boldsymbol{z}-I_{h} \boldsymbol{z}\right)\right)_{\Omega}+\left(s-\Pi_{h} s, \nabla \cdot\left(\boldsymbol{u}-\tilde{\boldsymbol{u}}_{h}\right)\right)_{\Omega}-\left(p-\tilde{p}_{h}, \nabla \cdot\left(\boldsymbol{z}-I_{h} \boldsymbol{z}\right)\right)_{\Omega} \\
& \quad+\sum_{K \in \mathcal{T}_{h}} \frac{h_{K}^{2}}{8 \nu}\left(-\nu \Delta \boldsymbol{u}+\nabla\left(p-\tilde{p}_{h}\right),-\nabla \Pi_{h} s\right)_{K} \cdot
\end{aligned}
$$


We now proceed to combine Cauchy-Schwarz inequality, the definition of $h$-norms, 3.2, Theorems 4.1 and 4.2 (4.2), 4.3, $2.12,2.16,2.17)$, and $(2.19)$ to deduce that

$$
\begin{aligned}
& \left\|\boldsymbol{u}-\tilde{\boldsymbol{u}}_{h}\right\|_{\boldsymbol{L}^{2}(\Omega)}^{2} \leq C\left(\nu\left|\boldsymbol{u}-\tilde{\boldsymbol{u}}_{h}\right|_{\boldsymbol{H}^{1}(\Omega)}^{2}+\left\|\nabla \cdot\left(\boldsymbol{u}-\tilde{\boldsymbol{u}}_{h}\right)\right\|_{\boldsymbol{L}^{2}(\Omega)}^{2}+\left\|p-\tilde{p}_{h}\right\|_{L^{2}(\Omega)}^{2}\right. \\
& \left.+\sum_{K \in \mathcal{T}_{h}} \frac{h_{K}^{2}}{8 \nu}\left\|-\nu \Delta \boldsymbol{u}+\nabla\left(p-\tilde{p}_{h}\right)\right\|_{\boldsymbol{L}^{2}(K)}^{2}+\sum_{F \in \mathcal{E}_{h}^{\text {int }}} \frac{h_{F}}{12 \nu}\left\|\llbracket \nu \partial_{\boldsymbol{n}} \mathcal{P}_{h}\left(\boldsymbol{u}-\tilde{\boldsymbol{u}}_{h}\right) \rrbracket_{F}\right\|_{\boldsymbol{L}^{2}(F)}^{2}\right)^{1 / 2} \\
& \times\left(\nu\left|\boldsymbol{z}-I_{h} \boldsymbol{z}\right|_{\boldsymbol{H}^{1}(\Omega)}^{2}+\left\|s-\Pi_{h} s\right\|_{L^{2}(\Omega)}^{2}+\sum_{K \in \mathcal{T}_{h}} \frac{h_{K}^{2}}{8 \nu}\left\|\nabla \Pi_{h} s\right\|_{\boldsymbol{L}^{2}(K)}^{2}+\left\|\nabla \cdot\left(\boldsymbol{z}-I_{h} \boldsymbol{z}\right)\right\|_{\boldsymbol{L}^{2}(\Omega)}^{2}\right. \\
& \left.+\sum_{F \in \mathcal{E}_{h}^{\text {int }}} \frac{h_{F}}{12 \nu}\left[\left\|\llbracket \nu \partial_{\boldsymbol{n}} \mathcal{P}_{h}\left(\boldsymbol{z}-I_{h} \boldsymbol{z}\right) \rrbracket_{F}\right\|_{\boldsymbol{L}^{2}(F)}^{2}+\left\|\llbracket \nu \partial_{\boldsymbol{n}} \mathcal{P}_{h} \boldsymbol{z} \rrbracket_{F}\right\|_{\boldsymbol{L}^{2}(F)}^{2}\right]\right)^{1 / 2} \\
& \leq C\left(\left\|\boldsymbol{u}-\tilde{\boldsymbol{u}}_{h}\right\|_{h}^{2}+\left\|p-\tilde{p}_{h}\right\|_{h}^{2}+\nu h^{2}|\boldsymbol{u}|_{\boldsymbol{H}^{2}(\Omega)}^{2}+\left\|p-\tilde{p}_{h}\right\|_{L^{2}(\Omega)}^{2}\right)^{1 / 2}\left(\left\|\boldsymbol{z}-I_{h} \boldsymbol{z}\right\|_{h}^{2}\right. \\
& \left.+\left\|s-\Pi_{h} s\right\|_{L^{2}(\Omega)}^{2}+h^{2}|\boldsymbol{z}|_{\boldsymbol{H}^{2}(\Omega)}^{2}+h^{2}\left|I_{h} \boldsymbol{z}\right|_{\boldsymbol{H}^{2}(\Omega)}^{2}+\sum_{K \in \mathcal{T}_{h}} \frac{h_{K}^{2}}{8 \nu}\left|\Pi_{h} s\right|_{\boldsymbol{H}^{1}(K)}^{2}\right)^{1 / 2} \\
& \leq C h^{2}\left(|\boldsymbol{u}|_{\boldsymbol{H}^{2}(\Omega)}+|p|_{H^{1}(\Omega)}\right)\left(h^{2}\left[|\boldsymbol{z}|_{\boldsymbol{H}^{2}(\Omega)}^{2}+|s|_{H^{1}(\Omega)}^{2}+\|s\|_{h}^{2}\right]\right)^{1 / 2} \\
& \leq C h^{2}\left(|\boldsymbol{u}|_{\boldsymbol{H}^{2}(\Omega)}+|p|_{H^{1}(\Omega)}\right)\left\|\boldsymbol{u}-\tilde{\boldsymbol{u}}_{h}\right\|_{\boldsymbol{L}^{2}(\Omega)},
\end{aligned}
$$

and the proof is complete after dividing by the last term in the RHS.

It is easily seen that using the local trace inequality 2.10 and Céa's lemma, it is possible to modify the regularity hypothesis of Theorems 4.2 and 4.3 setting $(\boldsymbol{u}, p) \in\left[\boldsymbol{H}^{1+\delta}(\Omega) \cap \boldsymbol{H}_{0}^{1}(\Omega)\right] \times\left[H^{\delta}(\Omega) \cap L_{0}^{2}(\Omega)\right]$, $1 / 2<\delta \leq 1$, to obtain the estimate

$$
\left\|\boldsymbol{u}-\tilde{\boldsymbol{u}}_{h}\right\|_{\boldsymbol{L}^{2}(\Omega)}+h^{\delta}\left\|p-\tilde{p}_{h}\right\|_{L^{2}(\Omega)} \leq C h^{1+\delta}\left(|\boldsymbol{u}|_{\boldsymbol{H}^{2}(\Omega)}+|p|_{H^{1}(\Omega)}\right) .
$$

\section{Superconvergence Analysis}

As briefly mentioned in Section 1, the present approach for establishing superconvergence estimates basically consists in projecting the FVE approximation $\left(\tilde{\boldsymbol{u}}_{h}, \tilde{p}_{h}\right) \in\left(\mathcal{V}_{h} \times Q_{h}\right)$ into a different finite dimensional space $\left(\mathcal{V}_{\rho}^{r} \times Q_{\rho}^{t}\right), r, t \geq 0$, which corresponds to the (possibly of higher order) counterpart of $\left(\mathcal{V}_{h} \times Q_{h}\right)$ associated to a coarser mesh $\mathcal{T}_{\rho}$ of size $\rho=h^{\alpha}$, with $\alpha \in(0,1)$.

We start by defining the operators $\Pi_{\rho}^{\mathcal{V}}, \Pi_{\rho}^{Q}$ as the $L^{2}$-projections onto $\mathcal{V}_{\rho}^{r}$ and $Q_{\rho}^{t}$ respectively. Therefore, in particular it holds that

$$
\begin{aligned}
\left\|\boldsymbol{v}-\Pi_{\rho}^{\mathcal{V}} \boldsymbol{v}\right\|_{\boldsymbol{L}^{2}(\Omega)} & \leq C \rho^{s}|\boldsymbol{v}|_{\boldsymbol{H}^{s}(\Omega)} & & 0 \leq s \leq r+1 \\
\left\|\Pi_{\rho}^{\mathcal{V}} \boldsymbol{v}\right\|_{\boldsymbol{L}^{2}(\Omega)} & \leq C\|\boldsymbol{v}\|_{\boldsymbol{L}^{2}(\Omega)}, & & \\
\left\|q-\Pi_{\rho}^{Q} q\right\|_{L^{2}(\Omega)} & \leq C \rho^{s}|q|_{H^{s}(\Omega)} & & 0 \leq s \leq t+1 .
\end{aligned}
$$

Let us also denote by $I_{\rho}$ the Lagrange interpolator into $\mathcal{V}_{\rho}^{1}$, and note that by 2.12 , (5.1) and the inverse inequality (see e.g. [19])

$$
\left\|v_{\rho}\right\|_{\boldsymbol{H}^{m}(K)} \leq C \rho^{-m}\left\|v_{\rho}\right\|_{\boldsymbol{L}^{2}(K)} \quad v_{\rho} \in \mathcal{V}_{\rho}^{r}, K \in \mathcal{T}_{\rho}
$$


it follows that

$$
\begin{aligned}
\left|\boldsymbol{v}-\Pi_{\rho}^{\mathcal{V}} \boldsymbol{v}\right|_{\boldsymbol{H}^{1}(\Omega)} & \leq\left|\boldsymbol{v}-I_{\rho} \boldsymbol{v}\right|_{\boldsymbol{H}^{1}(\Omega)}+\left|I_{\rho} \boldsymbol{v}-\Pi_{\rho}^{\mathcal{V}} \boldsymbol{v}\right|_{\boldsymbol{H}^{1}(\Omega)} \\
& \leq C\left(\rho^{s-1}|\boldsymbol{v}|_{\boldsymbol{H}^{s}(\Omega)}+\rho^{-1}\left\|I_{\rho} \boldsymbol{v}-\Pi_{\rho}^{\mathcal{V}} \boldsymbol{v}\right\|_{\boldsymbol{L}^{2}(\Omega)}\right) \\
& \leq C \rho^{s-1}|\boldsymbol{v}|_{\boldsymbol{H}^{s}(\Omega)}=C h^{\alpha(s-1)}|\boldsymbol{v}|_{\boldsymbol{H}^{s}(\Omega)} \quad 0 \leq s \leq r+1
\end{aligned}
$$

When considering particularly simple domains, it is also possible to handle a different choice for $\left(\mathcal{V}_{\rho}^{r} \times Q_{\rho}^{t}\right)$, such as B-splines or trigonometric functions as mentioned in [19. In that case, (5.1)- 5.3 should be properly rewritten.

Theorem 5.1 (Superconvergence for the velocity). Let $(\boldsymbol{u}, p) \in\left[\boldsymbol{H}^{2}(\Omega) \cap \boldsymbol{H}_{0}^{1}(\Omega)\right] \times\left[H^{1}(\Omega) \cap L_{0}^{2}(\Omega)\right]$ and $\left(\tilde{\boldsymbol{u}}_{h}, \tilde{p}_{h}\right) \in \mathcal{V}_{h} \times Q_{h}$ be the solutions of 2.4 and $(3.10$, respectively. Then there exists a positive constant C such that

$$
\begin{aligned}
& \left\|\boldsymbol{u}-\Pi_{\rho}^{\mathcal{V}} \tilde{\boldsymbol{u}}_{h}\right\|_{\boldsymbol{L}^{2}(\Omega)} \leq C h^{\alpha s}|\boldsymbol{u}|_{\boldsymbol{H}^{s}(\Omega)}+C h^{2}\left(|\boldsymbol{u}|_{\boldsymbol{H}^{2}(\Omega)}+|p|_{H^{1}(\Omega)}\right) \\
& \left|\boldsymbol{u}-\Pi_{\rho}^{\mathcal{V}} \tilde{\boldsymbol{u}}_{h}\right|_{\boldsymbol{H}^{1}(\Omega)} \leq C h^{\alpha(s-1)}|\boldsymbol{u}|_{\boldsymbol{H}^{s}(\Omega)}+C h^{2-\alpha}\left(|\boldsymbol{u}|_{\boldsymbol{H}^{2}(\Omega)}+|p|_{H^{1}(\Omega)}\right)
\end{aligned}
$$

for $0 \leq s \leq r+1$

Proof. By triangular inequality, 5.1 and definition of $\Pi_{\rho}^{\mathcal{V}}$ and $\rho$, it follows that

$$
\begin{aligned}
\left\|\boldsymbol{u}-\Pi_{\rho}^{\mathcal{V}} \tilde{\boldsymbol{u}}_{h}\right\|_{\boldsymbol{L}^{2}(\Omega)} & \leq\left\|\boldsymbol{u}-\Pi_{\rho}^{\mathcal{V}} \boldsymbol{u}\right\|_{\boldsymbol{L}^{2}(\Omega)}+\left\|\Pi_{\rho}^{\mathcal{V}}\left(\boldsymbol{u}-\tilde{\boldsymbol{u}}_{h}\right)\right\|_{\boldsymbol{L}^{2}(\Omega)} \\
& \leq C h^{\alpha s}|\boldsymbol{u}|_{\boldsymbol{H}^{s}(\Omega)}+\left\|\Pi_{\rho}^{\mathcal{V}}\left(\boldsymbol{u}-\tilde{\boldsymbol{u}}_{h}\right)\right\|_{\boldsymbol{L}^{2}(\Omega)} .
\end{aligned}
$$

The task now consists in estimating the second term in the RHS of $(5.6)$. First, note that since $\Pi_{\rho}^{\mathcal{V}}$ is a $L^{2}-$ projection, it satisfies

$$
\left(\Pi_{\rho}^{\mathcal{V}}\left(\boldsymbol{u}-\tilde{\boldsymbol{u}}_{h}\right), \boldsymbol{w}\right)_{\Omega}=\left(\boldsymbol{u}-\tilde{\boldsymbol{u}}_{h}, \Pi_{\rho}^{\mathcal{V}} \boldsymbol{w}\right)_{\Omega} \quad \forall \boldsymbol{w} \in \boldsymbol{L}^{2}(\Omega)
$$

A combination of this relation with norm properties gives

$$
\left\|\Pi_{\rho}^{\mathcal{V}}\left(\boldsymbol{u}-\tilde{\boldsymbol{u}}_{h}\right)\right\|_{\boldsymbol{L}^{2}(\Omega)}=\sup _{\boldsymbol{w} \in \boldsymbol{L}^{2}(\Omega) \backslash\{\mathbf{0}\}} \frac{\left|\left(\boldsymbol{u}-\tilde{\boldsymbol{u}}_{h}, \Pi_{\rho}^{\mathcal{V}} \boldsymbol{w}\right)_{\Omega}\right|}{\|\boldsymbol{w}\|_{\boldsymbol{L}^{2}(\Omega)}} .
$$

We now proceed to use 2.18 with the particular choices $\boldsymbol{\varphi}=\Pi_{\rho}^{\mathcal{V}} \boldsymbol{w}$ for some fixed $\boldsymbol{w} \in \boldsymbol{L}^{2}(\Omega)$, and $(\boldsymbol{v}, q)=\left(\boldsymbol{u}-\tilde{\boldsymbol{u}}_{h}, p-\tilde{p}_{h}\right)$. Thus, estimates 2.19) and 5.2 imply that

$$
|\boldsymbol{z}|_{\boldsymbol{H}^{2}(\Omega)}+|s|_{H^{1}(\Omega)} \leq C\|\boldsymbol{w}\|_{\boldsymbol{L}^{2}(\Omega)}
$$

where $(\boldsymbol{z}, s)$ is the solution of 2.18 . Therefore, using Lemma 4.1 with the choice $\left(\boldsymbol{v}_{h}, q_{h}\right)=\left(I_{h} \boldsymbol{z}, \Pi_{h} s\right) \in$ $\mathcal{V}_{h} \times Q_{h}$, gives

$$
\begin{aligned}
\left(\boldsymbol{u}-\tilde{\boldsymbol{u}}_{h}, \Pi_{\rho}^{\mathcal{V}} \boldsymbol{w}\right)_{\Omega} & =\nu\left(\nabla\left(\boldsymbol{u}-\tilde{\boldsymbol{u}}_{h}\right), \nabla\left(\boldsymbol{z}-I_{h} \boldsymbol{z}\right)\right)_{\Omega}+\left(s-\Pi_{h} s, \nabla \cdot\left(\boldsymbol{u}-\tilde{\boldsymbol{u}}_{h}\right)\right)_{\Omega}-\left(p-\tilde{p}_{h}, \nabla \cdot\left(\boldsymbol{z}-I_{h} \boldsymbol{z}\right)\right)_{\Omega} \\
& -\sum_{K \in \mathcal{T}_{h}} \frac{h_{K}^{2}}{8 \nu}\left(-\nu \Delta \boldsymbol{u}+\nabla\left(p-\tilde{p}_{h}\right), \nabla \Pi_{h} s\right)_{K}-\sum_{F \in \mathcal{E}_{h}^{\text {int }}} \frac{h_{F}}{12 \nu}\left(\llbracket \nu \partial_{\boldsymbol{n}} \mathcal{P}_{h}\left(\boldsymbol{u}-\tilde{\boldsymbol{u}}_{h}\right) \rrbracket_{F}, \llbracket \nu \partial_{\boldsymbol{n}} \mathcal{P}_{h} \boldsymbol{z} \rrbracket_{F}\right)_{F} .
\end{aligned}
$$

Then, from Cauchy inequality, 2.12, 2.17, 2.10, Theorems 4.1, 4.2, and estimates (5.8, 5.2 we obtain

$$
\begin{aligned}
\left(\boldsymbol{u}-\tilde{\boldsymbol{u}}_{h}, \Pi_{\rho}^{\mathcal{V}} \boldsymbol{w}\right)_{\Omega} \leq & \nu\left|\boldsymbol{u}-\tilde{\boldsymbol{u}}_{h}\right|_{\boldsymbol{H}^{1}(\Omega)}\left|\boldsymbol{z}-I_{h} \boldsymbol{z}\right|_{\boldsymbol{H}^{1}(\Omega)}+\left\|s-\Pi_{h} s\right\|_{L^{2}(\Omega)}\left|\boldsymbol{u}-\tilde{\boldsymbol{u}}_{h}\right|_{\boldsymbol{H}^{1}(\Omega)} \\
& +\left\|p-\tilde{p}_{h}\right\|_{L^{2}(\Omega)}\left|\boldsymbol{z}-I_{h} \boldsymbol{z}\right|_{\boldsymbol{H}^{1}(\Omega)}+\sum_{K \in \mathcal{T}_{h}} \frac{h_{K}^{2}}{8 \nu}\left\|-\nu \Delta \boldsymbol{u}+\nabla\left(p-\tilde{p}_{h}\right)\right\|_{\boldsymbol{L}^{2}(K)}\left\|\nabla \Pi_{h} s\right\|_{\boldsymbol{L}^{2}(K)} \\
& +\sum_{F \in \mathcal{E}_{h}^{\text {int }}} \frac{h_{F}}{12 \nu}\left\|\llbracket \nu \partial_{\boldsymbol{n}} \mathcal{P}_{h}\left(\boldsymbol{u}-\tilde{\boldsymbol{u}}_{h}\right) \rrbracket_{F}\right\|_{\boldsymbol{L}^{2}(F)}\left\|\llbracket \nu \partial_{\boldsymbol{n}} \mathcal{P}_{h} \boldsymbol{z} \rrbracket_{F}\right\|_{\boldsymbol{L}^{2}(F)}
\end{aligned}
$$




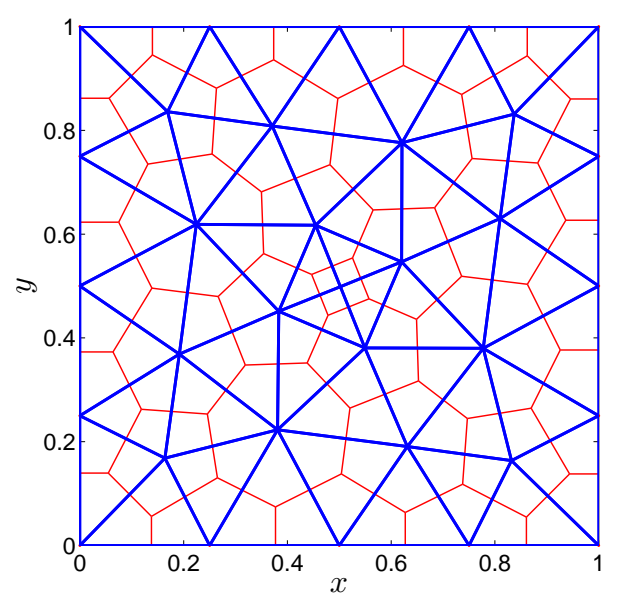

Figure 4. Example of primal and dual meshes $\mathcal{T}_{h}, \mathcal{T}_{h}^{\star}$ on $\Omega=(0,1)^{2}$ (17 interior nodes).

$$
\begin{aligned}
\leq & \left(2 \nu\left|\boldsymbol{u}-\tilde{\boldsymbol{u}}_{h}\right|_{\boldsymbol{H}^{1}(\Omega)}^{2}+\frac{1}{\nu}\left\|p-\tilde{p}_{h}\right\|_{L^{2}(\Omega)}^{2}+\sum_{K \in \mathcal{T}_{h}} \frac{h_{K}^{2}}{8}\|\Delta \boldsymbol{u}\|_{\boldsymbol{L}^{2}(K)}^{2}\right. \\
& \left.+\sum_{K \in \mathcal{T}_{h}} \frac{h_{K}^{2}}{8 \nu}\left|p-\tilde{p}_{h}\right|_{H^{1}(K)}^{2}+\sum_{F \in \mathcal{E}_{h}^{\text {int }}} \frac{h_{F}}{12 \nu}\left\|\llbracket \nu \partial_{\boldsymbol{n}} \mathcal{P}_{h}\left(\boldsymbol{u}-\tilde{\boldsymbol{u}}_{h}\right) \rrbracket_{F}\right\|_{\boldsymbol{L}^{2}(F)}^{2}\right)^{1 / 2} \\
& \times\left(2 \nu\left|\boldsymbol{z}-I_{h} \boldsymbol{z}\right|_{\boldsymbol{H}^{1}(\Omega)}^{2}+\frac{1}{\nu}\left\|s-\Pi_{h} s\right\|_{L^{2}(\Omega)}^{2}+\sum_{F \in \mathcal{E}_{h}^{\text {int }}} \frac{h_{F}}{12 \nu}\left\|\llbracket \nu \partial_{\boldsymbol{n}} \mathcal{P}_{h} I_{h} \boldsymbol{z} \rrbracket_{F}\right\|_{\boldsymbol{L}^{2}(F)}^{2}\right. \\
& \left.+\sum_{K \in \mathcal{T}_{h}} \frac{h_{K}^{2}}{8 \nu}\left\|\nabla \Pi_{h} s\right\|_{\boldsymbol{L}^{2}(K)}^{2}\right)^{1 / 2} \\
\leq & C\left(\left\|\boldsymbol{u}-\tilde{\boldsymbol{u}}_{h}\right\|_{h}^{2}+\left\|p-\tilde{p}_{h}\right\|_{h}^{2}+\frac{1}{\nu}\left\|p-\tilde{p}_{h}\right\|_{L^{2}(\Omega)}^{2}+\nu h^{2}|\boldsymbol{u}|_{\boldsymbol{H}^{2}(\Omega)}^{2}\right)^{1 / 2} \\
& \times\left((\nu+1) h^{2}|\boldsymbol{z}|_{\boldsymbol{H}^{2}(\Omega)}^{2}+h^{2} \frac{(\nu+1)}{\nu}|s|_{H^{1}(\Omega)}^{2}\right)^{1 / 2} \\
\leq & C h^{2}\left(|\boldsymbol{u}|_{\boldsymbol{H}^{2}(\Omega)}+|p|_{H^{1}(\Omega)}\right)\|\boldsymbol{w}\|_{\boldsymbol{L}^{2}(\Omega)} .
\end{aligned}
$$

Finally, by applying (5.6) and (5.7) the proof of the $L^{2}$-estimate is completed. For the second estimate, it suffices to use a similar argument combined with (5.5).

Combining (2.10), (5.4), (5.5) and the definition of the $h$-norm, we can also conclude that the next result holds.

Corollary 5.1. Under the hypotheses of Theorem 5.1, we have the following estimate

$$
\left\|\boldsymbol{u}-\Pi_{\rho}^{\mathcal{V}} \tilde{\boldsymbol{u}}_{h}\right\|_{h}^{2} \leq C h^{\alpha(s-1)}\left(h+h^{\alpha}+1\right)|\boldsymbol{u}|_{H^{s}(\Omega)}+C h^{2}\left(h^{1-\alpha}+h^{-\alpha}+1\right)\left(|\boldsymbol{u}|_{H^{2}(\Omega)}+|p|_{H^{1}(\Omega)}\right),
$$

for $0 \leq s \leq r+1$.

Remark 5.1. We stress that Theorem 5.1 does not provide an improvement of the convergence rate for the velocity field in the $L^{2}$-norm in the studied case of $\mathbb{P}_{1}$ elements. This holds even if in the postprocessing stage we use a different space for the velocity such as $\mathbb{P}_{r}, r \geq 2$. However the superconvergence is achieved in the $\boldsymbol{H}^{1}$-seminorm for $\mathbb{P}_{r}, r \geq 2$ and for $\alpha>1 / 2$. The following result (which may be proved in much 


\begin{tabular}{ccccccc}
\hline$N_{h}$ & $e(\boldsymbol{u})$ & $r(\boldsymbol{u})$ & $e(p)$ & $r(p)$ & $E(\boldsymbol{u}, p)$ & $R(\boldsymbol{u}, p)$ \\
\hline 121 & $1.9446 \times 10^{-3}$ & - & $5.7385 \times 10^{-3}$ & - & $7.0973 \times 10^{-2}$ & - \\
449 & $4.8838 \times 10^{-4}$ & 1.9920 & $2.6121 \times 10^{-3}$ & 1.1051 & $3.5083 \times 10^{-3}$ & 1.0208 \\
1729 & $1.2265 \times 10^{-4}$ & 1.9981 & $1.3041 \times 10^{-3}$ & 1.0740 & $1.6941 \times 10^{-3}$ & 1.0457 \\
6785 & $3.0518 \times 10^{-5}$ & 2.0015 & $6.1270 \times 10^{-4}$ & 1.0409 & $8.4207 \times 10^{-4}$ & 1.0114 \\
26881 & $7.5632 \times 10^{-6}$ & 2.0109 & $2.9861 \times 10^{-4}$ & 1.0372 & $4.1615 \times 10^{-4}$ & 1.0221 \\
107009 & $1.8847 \times 10^{-6}$ & 2.0117 & $1.4684 \times 10^{-4}$ & 1.0212 & $2.1062 \times 10^{-4}$ & 1.0093 \\
\hline
\end{tabular}

TABLE 1. Degrees of freedom $N_{h}$, computed errors and observed convergence rates.

the same way as Theorem 5.1, by using a duality argument) yields superconvergence for the pressure field as well, even in the case $t=1$.

Theorem 5.2 (Superconvergence for the pressure). Assume that $(\boldsymbol{u}, p) \in\left[\boldsymbol{H}^{2}(\Omega) \cap \boldsymbol{H}_{0}^{1}(\Omega)\right] \times\left[H^{1}(\Omega) \cap L_{0}^{2}(\Omega)\right]$ and $\left(\tilde{\boldsymbol{u}}_{h}, \tilde{p}_{h}\right) \in \mathcal{V}_{h} \times Q_{h}$ are the solutions of (2.4) and (3.10) respectively. Then there exists a positive constant C such that

$$
\left\|p-\Pi_{\rho}^{Q} \tilde{p}_{h}\right\|_{\boldsymbol{L}^{2}(\Omega)} \leq C h^{\alpha s}|p|_{H^{s}(\Omega)}+C h^{2-\alpha}\left(|\boldsymbol{u}|_{\boldsymbol{H}^{2}(\Omega)}+|p|_{H^{1}(\Omega)}\right), \quad \alpha>1 / 2,0 \leq s \leq t+1 .
$$

Remark 5.2. As briefly mentioned at the end of Section 2.2. the derivation of $(2.9)$ requires $f$ to be piecewise constant. Notice however, that if $\boldsymbol{f} \in \boldsymbol{H}^{1}(\Omega)$, the relevant term appearing in the deduction of the error analysis for (3.10) (take for instance the proof of Theorem 4.3. and recall that we have taken $\left.\boldsymbol{\varphi}=\boldsymbol{u}-\tilde{\boldsymbol{u}}_{h}\right)$ is readily estimated as

$$
\begin{aligned}
\left(\boldsymbol{f}, I_{h} \boldsymbol{z}-\mathcal{P}_{h} I_{h} \boldsymbol{z}\right)_{\Omega} & =\left(\boldsymbol{f}-\Pi_{h} \boldsymbol{f}, I_{h} \boldsymbol{z}-\mathcal{P}_{h} I_{h} \boldsymbol{z}\right)_{\Omega} \\
& \leq\left\|\boldsymbol{f}-\Pi_{h} \boldsymbol{f}\right\|_{\boldsymbol{L}^{2}(\Omega)}\left\|I_{h} \boldsymbol{z}-\mathcal{P}_{h} I_{h} \boldsymbol{z}\right\|_{\boldsymbol{L}^{2}(\Omega)} \\
& \leq C h^{2}\|\boldsymbol{f}\|_{\boldsymbol{H}^{1}(\Omega)}\left|I_{h} \boldsymbol{z}\right|_{\boldsymbol{H}^{1}(\Omega)} \\
& \leq C h^{2}\|\boldsymbol{f}\|_{\boldsymbol{H}^{1}(\Omega)}\|\boldsymbol{\varphi}\|_{\boldsymbol{L}^{2}(\Omega)} \\
& =C h^{2}\|\boldsymbol{f}\|_{\boldsymbol{H}^{1}(\Omega)}\left\|\boldsymbol{u}-\tilde{\boldsymbol{u}}_{h}\right\|_{\boldsymbol{L}^{2}(\Omega)}
\end{aligned}
$$

where we have applied Lemma 3.1, properties of $I_{h}, \Pi_{h}$, and 2.19). Then, performing an analogous analysis to that presented in [2, Appendix B], it is possible to recast the estimate of Theorem 4.3 as

$$
\left\|\boldsymbol{u}-\tilde{\boldsymbol{u}}_{h}\right\|_{\boldsymbol{L}^{2}(\Omega)} \leq C h^{2}\left(|\boldsymbol{u}|_{\boldsymbol{H}^{2}(\Omega)}+|p|_{H^{1}(\Omega)}+\|\boldsymbol{f}\|_{\boldsymbol{H}^{1}(\Omega)}\right) .
$$

Analogously, it is not difficult to extend all our convergence and superconvergence results to cover the general case $\boldsymbol{f} \in \boldsymbol{L}^{2}(\Omega)$. In such case, the estimates are of the same order than those presented in the paper.

\section{A Numerical Test}

We present an example illustrating the performance of the proposed FVE scheme on a set of triangulations of the domain $\Omega=(0,1)^{2}$ (see Figure 4p. In the following, by $e(\boldsymbol{u}):=\left\|\boldsymbol{u}-\tilde{\boldsymbol{u}}_{h}\right\|_{\boldsymbol{L}^{2}(\Omega)}, e(p):=\left\|p-\tilde{p}_{h}\right\|_{L^{2}(\Omega)}$ and $E(\boldsymbol{u}, p):=\left\|\boldsymbol{u}-\tilde{\boldsymbol{u}}_{h}\right\|_{h}+\left\|p-\tilde{p}_{h}\right\|_{h}$ we will denote errors, and $r(\boldsymbol{u}), r(p)$ and $R(\boldsymbol{u}, p)$ will denote the experimental rates of convergence given by

$$
r(\boldsymbol{u})=\frac{\log (e(\boldsymbol{u}) / \hat{e}(\boldsymbol{u}))}{\log (h / \hat{h})}, \quad r(p)=\frac{\log (e(p) / \hat{e}(p))}{\log (h / \hat{h})}, \quad R(\boldsymbol{u}, p)=\frac{\log (E(\boldsymbol{u}, p) / \hat{E}(\boldsymbol{u}, p))}{\log (h / \hat{h})},
$$

where $e$ and $\hat{e}$ ( $E$ and $\hat{E}$ respectively) stand for the corresponding errors computed for two consecutive meshes of sizes $h$ and $\hat{h}$. In the implementation we have used a standard Uzawa algorithm (see e.g. [15]) with a stopping criterion of $\left\|\tilde{p}_{h}^{r}-\tilde{p}_{h}^{r+1}\right\|_{L^{2}(\Omega)} \leq 10^{-6}$. 

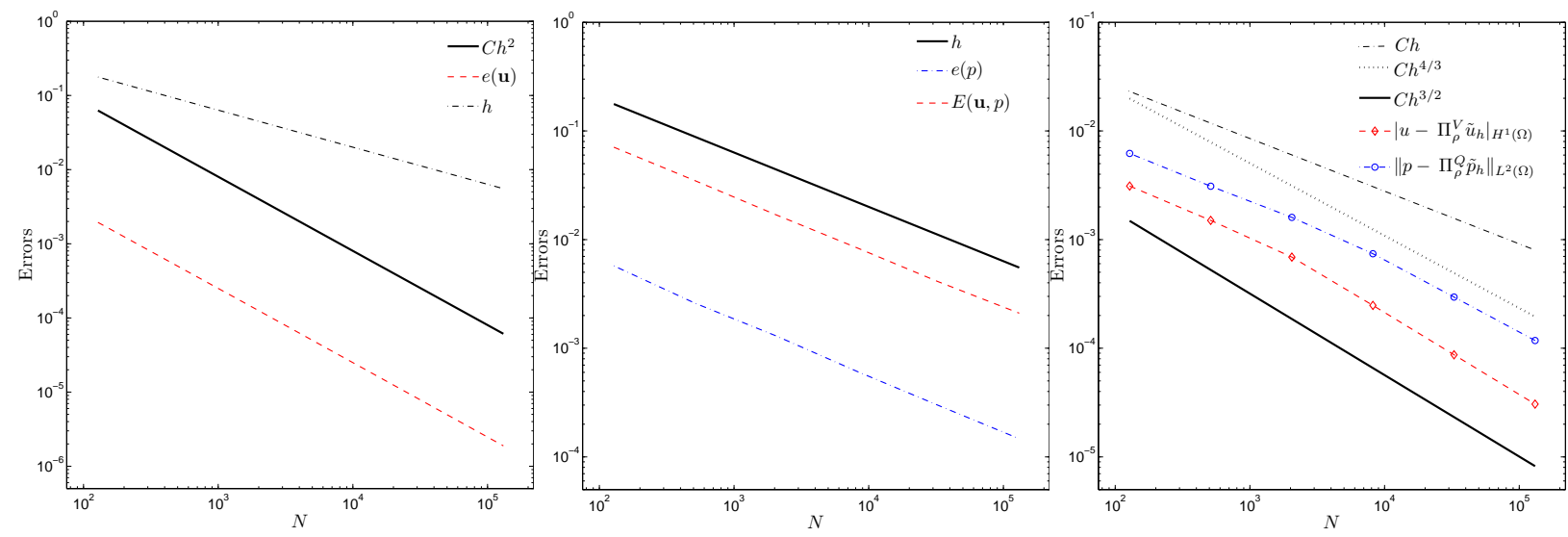

FiguRE 5. Left and middle plots: convergence history for the FVE method. Right plot: superconvergence rates obtained by a higher order postprocessing procedure.

We set $\nu=1$ and the forcing term $\boldsymbol{f}$ chosen in such a way that the exact solution of (2.1)-(2.3) is $\boldsymbol{u}=\left(\left(x_{1}^{4}-2 x_{1}^{3}+x_{1}^{2}\right)\left(4 x_{2}^{3}-6 x_{2}^{2}+2 x_{2}\right),-\left(4 x_{1}^{3}-6 x_{1}^{2}+2 x_{1}\right)\left(x_{2}^{4}-2 x_{2}^{3}+x_{2}^{2}\right)\right)^{T}, p(x)=x_{1}^{5}+x_{2}^{5}$. Notice that p satisfies $\int_{\Omega} p=0$ and $(\boldsymbol{u}, p)$ has a regular behaviour in the whole domain $\Omega$ (and then the regularity assumptions of Section 4 are satisfied). In Table 1 and Figure $5(a, b)$ we depict the convergence history of this example. The dominant error is $E(\boldsymbol{u}, p)$. More precisely, in $E(\boldsymbol{u}, p)$ the term $\left\|p-\tilde{p}_{h}\right\|_{h}$ is dominating, followed by $\left|\boldsymbol{u}-\tilde{\boldsymbol{u}}_{h}\right|_{\boldsymbol{H}^{1}(\Omega)}$. It is clearly seen that the rates of convergence $\mathcal{O}(h)$ and $\mathcal{O}\left(h^{2}\right)$ anticipated by Theorem 4.1. Theorem 4.2 and Theorem 4.3 are confirmed by the numerical results.

Now we apply a postprocessing technique by considering a coarser mesh of size $\rho=h^{2 / 3}$. Table 2 and Figure 5(c) show the superconvergence behavior of the approximate solution when a postprocessing algorithm with Taylor-Hood $\left(\mathbb{P}_{2}-\mathbb{P}_{1}\right)$ elements is applied. It is observed that as $h$ decreases, the convergence rate for the velocity approaches asymptotically $h^{3 / 2}$ and that of the pressure approaches $h^{4 / 3}$. This behavior is predicted by Theorems 5.1 and 5.2 with the setting $r=2, t=1$ and $\alpha=2 / 3$.

\section{Conclusion}

In this paper we have developed a FVE method for the Stokes problem. The discretization scheme is associated to a FE method in which a multiscale enhancement of the approximation space for the velocity field is performed. We have exploited some of the potential advantages of FVE discretizations with respect to classical finite volume methods, such as the flexibility in handling unstructured triangulations of complex geometries and that the discretization is constructed on the basis of the variational background of $\mathrm{FE}$ methods, therefore being more suitable to perform $L^{2}$-error analysis. This error analysis was performed for the case of piecewise linear continuous interpolation spaces only, nevertheless the same idea could be extended to a more general framework. A superconvergence analysis based on $L^{2}$-projections was also proposed, and the numerical experiments provided in this paper confirmed our theoretical findings. Finally we mention that extensions of this approach to other relevant problems, such as the generalized and transient Stokes problems, high-order approximation methods, and a posteriori error analysis are part of current and future work.

\section{Acknowledgments}

The authors wish to express their gratitude to Gabriel Barrenechea for his valuable suggestions and comments concerning the stabilized method. In addition, the authors would like to acknowledge financial 


\begin{tabular}{ccc|ccc}
\hline$N_{h}$ & $\left|\boldsymbol{u}-\Pi_{\rho}^{\nu} \tilde{\boldsymbol{u}}_{h}\right|_{\boldsymbol{H}^{1}(\Omega)}$ & rate & $\left\|p-\Pi_{\rho}^{Q} \tilde{p}_{h}\right\|_{L^{2}(\Omega)}$ & rate \\
\hline 121 & $3.1154 \times 10^{-3}$ & - & $6.2179 \times 10^{-3}$ & - \\
449 & $1.9147 \times 10^{-3}$ & 0.9011 & $3.1254 \times 10^{-3}$ & 1.0168 \\
1729 & $7.8238 \times 10^{-4}$ & 1.0815 & $1.6411 \times 10^{-3}$ & 0.9705 \\
6785 & $3.2394 \times 10^{-4}$ & 1.2755 & $7.4130 \times 10^{-4}$ & 1.0788 \\
26881 & $1.1455 \times 10^{-4}$ & 1.5124 & $2.9608 \times 10^{-4}$ & 1.3261 \\
107009 & $3.9812 \times 10^{-5}$ & 1.5076 & $1.1837 \times 10^{-4}$ & 1.3279 \\
\hline
\end{tabular}

TABLE 2. Degrees of freedom $N_{h}$, meshsizes $h$, computed errors and observed superconvergence rates. Postprocessing with Taylor-Hood elements and with the choice $\rho=h^{2 / 3}$.

support by the European Research Council through the Advanced Grant Mathcard, Mathematical Modelling and Simulation of the Cardiovascular System, Project ERC-2008-AdG 227058.

\section{REFERENCES}

[1] S. Agmon, Lectures on elliptic boundary value problems, AMS Providence, Rhode Island, 2010.

[2] R. Araya, G. Barrenechea and F. Valentin, Stabilized finite element methods based on multiscale enrichment for the Stokes problem. SIAM J. Numer. Anal. 44, 322-348 (2006)

[3] R.E. Bank and D.J. Rose, Some error estimates for the box method. SIAM J. Numer. Anal. 24, 777-787 (1987)

[4] C. Bernardi and G. Raugel, Méthodes d'éléments finis mixtes pour les équations de Stokes et de Navier-Stokes dans un polygone non convexe. Calcolo 18, 255-291 (1981)

[5] Ch. Bi and V. Ginting, A residual-type a posteriori error estimate of finite volume element method for a quasi-linear elliptic problem. Numer. Math. 114, 107-132 (2009)

[6] Z. Cai, On the finite volume element method. Numer. Math. 58, 713-735 (1991)

[7] S.C. Chou, Analysis and convergence of a covolume method for the generalized Stokes problem. Math. Comp. 66, 85-104 (1997)

[8] S.C. Chou and D.Y. Kwak, Multigrid algorithms for a vertex-centered covolume method for elliptic problems. Numer. Math. 90, 441-458 (2002)

[9] Ph. Clément, Approximation by finite element functions using local regularization. RAIRO Anal. Numer. 9, 77-84 (1975)

[10] M. Cui and X. Ye, Superconvergence of finite volume methods for the Stokes equations. Numer. Methods PDEs 25, $1212-1230(2009)$

[11] L. El Alaoui, An adaptive finite volume box scheme for solving a class of nonlinear parabolic equations. Appl. Math. Letters 22, 291-296 (2009)

[12] L. El Alaoui and A. Ern, Residual and hierarchical a posteriori error estimates for nonconforming mixed finite element methods. ESAIM: Math. Model. Numer. Anal. 38, 903-929 (2004)

[13] A. Ern and J.-L. Guermond, Theory and practice of finite elements, Springer-Verlag, New York, 2004.

[14] R. Eymard, R. Herbin and J.C. Latché, On a stabilized colocated finite volume scheme for the Stokes problem. ESAIM: Math. Model. Numer. Anal. 40, 501-527 (2006)

[15] M. Fortin and R. Glowinsky, Méthodes de lagrangien augmenté, Dunod, Paris, 1982.

[16] L. Franca, A. Madureira and F. Valentin, Towards multiscale functions: Enriching finite element spaces with local but not bubble-like functions, Comput. Methods Appl. Mech. Engrg. 194, 2077-2094 (2005)

[17] T. Gallouët, R. Herbin and J.C. Latché, A convergent finite element-finite volume scheme for the compressible Stokes problem. Part I: The isothermal case. Math. Comp. 78, 133-1352 (2009)

[18] V. Girault and P.A. Raviart, Finite element methods for Navier-Stokes equations, Springer-Verlag, Berlin, 1986.

[19] B.-O. Heimsund, X.-C. Tai and J. Wang, Superconvergence for the gradient of finite element approximations by $L^{2}$ projections. SIAM J. Numer. Anal. 40, 1263-1280 (2002)

[20] L. Ju, L. Tian and D. Wang, A posteriori error estimates for finite volume approximations of elliptic equations on general surfaces. Comput. Methods Appl. Mech. Engrg. 198, 716-726 (2009)

[21] B.P. Lamichhane, Inf-sup stable finite-element pairs based on dual meshes and bases for nearly incompressible elasticity. IMA J. Numer. Anal. 29, 404-420 (2009)

[22] J. Li, J. Wang and X. Ye, Superconvergence by $L^{2}$-projections for stabilized finite element methods for the Stokes equations. Int. J. Numer. Anal. Model. 6, 711-723 (2009)

[23] J. Li and Z. Chen, A new stabilized finite volume method for the stationary Stokes equations. Adv. Comput. Math. 30, $141-152(2009)$

[24] J. Li, Z. Chen and W. Wu, Generalized difference methods for differential equations: numerical analysis of finite volume methods, Marcel Dekker, New York, 2000. 
[25] S. Nicase and K. Djadel, Convergence analysis of a finite volume method for the Stokes system using non-conforming arguments. IMA J. Numer. Anal. 25 523-548 (2005)

[26] A. Quarteroni, Numerical models for differential problems. MS\&A series Vol. 2, Springer-Verlag, Milan, 2009.

[27] A. Quarteroni and A. Valli, Numerical approximation of partial differential equations. Springer-Verlag, Berlin, 1997.

[28] $\mathrm{H}$. Wu and R. Li, Error estimates for finite volume element methods for general second-order elliptic problems. Numer. Methods PDEs 19, 693-708 (2003)

[29] J. Wang and X. Ye, Superconvergence analysis for the Stokes problem by least squares surface fitting. SIAM J. Numer. Anal. 39 1001-1013 (2001)

[30] X. Ye, On the relationship between finite volume and finite element methods applied to the Stokes equations. Numer. Methods PDEs 5, 440-453 (2001)

[31] X. Ye, Superconvergence of nonconforming finite element methods for the Stokes equations. Numer. Methods PDEs 18, 143-154 (2002)

[32] X. Ye, A discontinuous finite volume method for the Stokes problems. SIAM J. Numer. Anal. 44, 183-198 (2006) 